\title{
THE VARIABLE STARS OF THE DRACO DWARF SPHEROIDAL GALAXY: REVISITED
}

\author{
K. Kinemuchi ${ }^{1,2}$, H. C. Harris ${ }^{3}$, Horace A. Smith $^{4}$, N. A. Silbermann ${ }^{5}$, L. A. Snyder ${ }^{4}$, A. P. LaCluyzé ${ }^{4}$, And C. L. Clark \\ ${ }^{1}$ Departamento de Astronomía, Universidad de Concepción, Concepción, Chile \\ 2 Department of Astronomy, University of Florida, Gainesville, Fl 32611, USA \\ ${ }^{3}$ US Naval Observatory, Flagstaff Station, Flagstaff, AZ 86001, USA \\ ${ }^{4}$ Department of Physics \& Astronomy, Michigan State University, East Lansing, MI 48824, USA \\ 5 Spitzer Science Center, Mail Stop 220-6, Pasadena, CA 91125, USA \\ Received 2007 October 12; accepted 2008 August 12; published 2008 October 9
}

\begin{abstract}
We present a CCD survey of variable stars in the Draco dwarf spheroidal galaxy. This survey, which has the largest areal coverage since the original variable star survey by Baade \& Swope, includes photometry for 270 RR Lyrae (RRL) stars, 9 anomalous Cepheids (ACs), 2 eclipsing binaries, and 12 slow, irregular red variables, as well as 30 background QSOs. Twenty-six probable double-mode RRL stars were identified. Observed parameters, including mean $V$ and $I$ magnitudes, $V$ amplitudes, and periods, have been derived. Photometric metallicities of the ab-type RRL stars were calculated according to the method of Jurcsik \& Kovacs, yielding a mean metallicity of $\langle[\mathrm{Fe} / \mathrm{H}]\rangle=-2.19 \pm 0.03$. The well-known Oosterhoff intermediate nature of the RRL stars in Draco is reconfirmed, although the double-mode RRL stars, with one exception, have properties similar to those found in Oosterhoff type II globular clusters. The period-luminosity relation of the ACs is rediscussed with the addition of the new Draco ACs.
\end{abstract}

Key words: galaxies: dwarf - stars: RR Lyrae - stars: variables: other

Online-only material: extended figure, machine-readable and VO table

\section{INTRODUCTION}

The Draco dwarf spheroidal $(\mathrm{dSph})$ galaxy $\left(\alpha_{2000.0}=\right.$ $\left.17^{\mathrm{h}} 20^{\mathrm{m}} 12^{\mathrm{s}} .39, \delta_{2000.0}=+57^{\circ} 54^{\prime} 55^{\prime \prime} .3\right)$, a satellite of the Milky Way, was first extensively studied by Baade \& Swope (1961) (hereafter known as B\&S). They reported discovering over 260 variable stars and obtained photometry for 138 variables in the central region of Draco, 133 of which were of RR Lyrae (RRL) type. Several subsequent studies have investigated aspects of the variable star population in Draco. Zinn \& Searle (1976) reported new observations of the anomalous Cepheids (ACs) in Draco. Nemec (1985a) re-analyzed the B\&S photometry and produced updated periods for the $B \& S$ variables. Both Nemec (1985a) and Goranskij (1982) reported new doublemode RRL stars in Draco. Recently, Bonanos et al. (2004) provided a photometric study of Draco which produced light curves for 146 RRL stars, four ACs, an SX Phe star, and a field eclipsing binary. In this work, we use CCD observations to update the census of variable stars in Draco. We cover an area slightly larger than the full B\&S survey, and we discover new variables with smaller amplitudes than those found by B\&S. We provide photometric data, periods, and light curves for over 320 variable stars.

This paper is organized as follows: Section 2 describes our data acquisition and data reduction processes. Section 3 covers our analysis techniques. Periods, light curves, and classifications of the variable stars are presented in Section 4. A rediscussion of the Oosterhoff classification of the Draco dSph is presented in Section 5. Conclusions are summarized in Section 6.

\section{DATA ACQUISITION AND REDUCTION}

Our survey of the Draco dSph galaxy was conducted at two telescopes: the $1.0 \mathrm{~m}$ at the US Naval Observatory (USNO) in Flagstaff, AZ, and the $2.3 \mathrm{~m}$ telescope at the Wyoming Infrared Observatory (WIRO), at Mt. Jelm, Wyoming. Combined, the two data sets cover a time interval of four years (1993-1996).
Table 1 contains the Heliocentric Julian Dates for when the data were observed.

\subsection{USNO Observations}

Images of Draco were taken with the $1.0 \mathrm{~m}$ telescope of USNO in Flagstaff, AZ, during the 1995 and 1996 observing seasons. A Tektronix $2048 \times 2048$ CCD was used with a pixel size of $00^{\prime \prime} 68$, giving a field size of 23.2 . Four fields were observed, each covering one quadrant of Draco, with $1^{\prime}$ overlap between fields, thus covering approximately a square region of $45^{\prime}$ size centered on Draco. The northeast field position was shifted to the east to avoid the bright star just north of Draco. Therefore, the northeast and northwest fields did not overlap, and three variable stars (V5, V10, and V117) were missed in this narrow gap. Figure 1 shows the field placement. This areal coverage is larger than any other study of the variable stars in Draco-it covers about four times more area and more than twice the number of variable stars than the study by Bonanos et al. (2004), and it provides a useful coverage of about two times more area than that of B\&S. The Palomar 200 inch telescope used by B\&S allowed discovery of some variable stars up to a distance of $24^{\prime}$ from the center of Draco. However, the degraded image quality in the outer parts of their field prevented them from measuring magnitudes or deriving light curves and periods for most variables beyond an $8^{\prime}$ radius from Draco. This coverage includes all known variable stars in Draco from the B\&S study except for two stars they identified, which were found at large distances from the galaxy (one far east, V205, and the other far west, V333). Also missing from our study are the three stars that lie in the gap between the northeast and northwest fields near the bright star on the north side of Draco. The first part of Table 2 lists these stars.

The images were taken with a Johnson $V$ filter throughout the 1995 and 1996 observing seasons and with a Cousins $I$ filter mostly during the 1996 season. The seeing was typically $2^{\prime \prime}$, and the exposure times were 15-30 minutes depending on the 


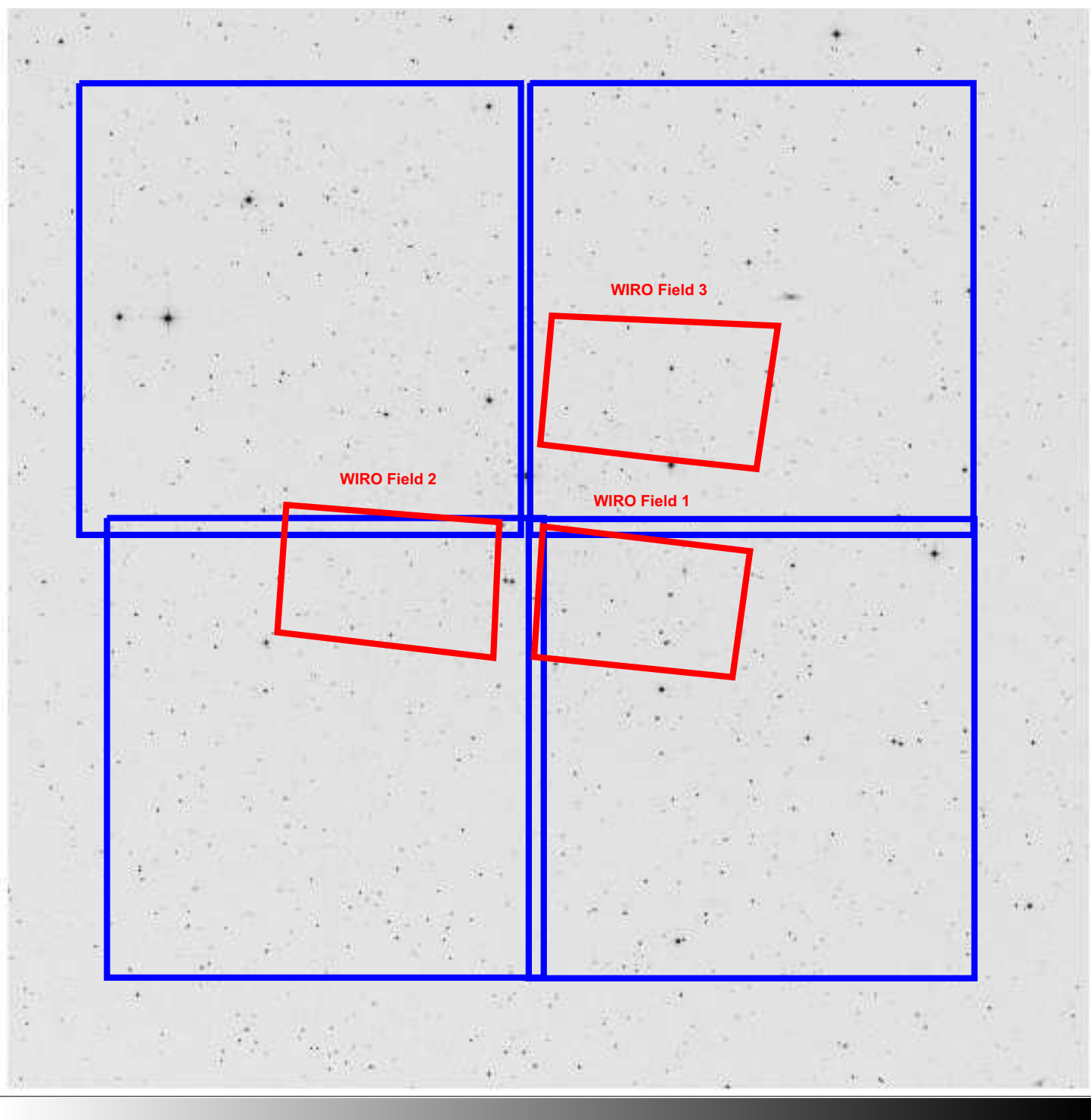

Figure 1. Observed fields of the Draco dSph galaxy. The regions outlined in blue are fields observed at USNO, while the red boxes are the fields observed at WIRO.

Table 1

Observations of Draco Dwarf Galaxy in Heliocentric Julian Date (2449000.0 + Days)

\begin{tabular}{ccc}
\hline \hline Year & USNO & WIRO \\
\hline 1993 & $\ldots$ & $183-187$ \\
1994 & $424-427$ & $\ldots$ \\
& $453-456$ & $\ldots$ \\
& $463-464$ & $\ldots$ \\
& 482 & $\ldots$ \\
& 501 & $\ldots$ \\
& 512 & $511-513$ \\
& $541-544$ & $\ldots$ \\
& 560 & $\ldots$ \\
& $597-600$ & $\ldots$ \\
& $629-630$ & $\ldots$ \\
& $804-805$ & $\ldots$ \\
& $833-836$ & $\ldots$ \\
& $892-893$ & $\ldots$ \\
& $923-926$ & $\ldots$ \\
\hline
\end{tabular}

seeing. Exposures were taken switching between quadrants, and alternating filters in 1996, so that each quadrant was observed
1-4 times on a given night with a given filter. An effort was made to minimize alias effects by observing each quadrant over $6-8 \mathrm{hr}$ on several nights, by observing over three weeks during several months, and by observing over the full range of months possible each season. A total of 39-41 V images and 19-20 I images of each quadrant were taken and are included in the following analysis. DAOPHOT (Stetson 1987) was used to measure all images. A small radial correction for image distortion in the corners of each image was applied for the data taken at USNO.

For the goals of identifying variable stars and measuring accurate magnitudes and light curves, five sources contribute errors to these data. Some stars are crowded or near brighter stars and have erroneous measurements. The CCD has a few defects that produce spurious magnitudes for some stars that occasionally fall on a defect. The CCD is not physically flat, so the high center and low corners produce images of stars in the corners of the field that are not in perfect focustogether with astigmatism, the resulting magnitudes have some additional error. The desire for short exposure times has resulted in images that have typically 0.03 mag error for each observation for the RRL and other horizontal-branch (HB) stars in Draco. Finally, the inevitable cosmic rays occasionally affect a star 
Table 2

Stars in the Draco Field Not Observed or Found Not to be Variable

\begin{tabular}{lccc}
\hline \hline B\&S ID & $\begin{array}{c}\text { R.A. } \\
(2000.0)\end{array}$ & $\begin{array}{c}\text { Decl. } \\
(2000.0)\end{array}$ & Comments \\
\hline V333 & $17: 17: 24.0$ & $58: 02: 32.0$ & West of USNO fields \\
V5 & $17: 20: 20.4$ & $58: 00: 55.0$ & In the gap between quadrants 1 and $4(P=0.57006$ days $)$ \\
V117 & $17: 20: 21.1$ & $58: 05: 43.0$ & In the gap between quadrants 1 and 4 , not measured by B\&S \\
V10 & $17: 20: 23.9$ & $57: 58: 44.0$ & In the gap between quadrants 1 and 4, \\
& & & near bright star, not measured by B\&S \\
V205 & $17: 23: 19.6$ & $57: 57: 55.0$ & East of USNO fields \\
Nonvariable Stars & & & \\
V168 & & $58: 00: 35.0$ & Outside central field. Not measured by B\&S \\
V195 & $17: 19: 27.24$ & $57: 52: 58.7$ & Near bright star. Not measured by B\&S \\
V111 & $17: 20: 27.61$ & $57: 52: 58.9$ & Near bright star. Not measured by B\&S \\
\hline
\end{tabular}

Table 3

Transformation Coefficients for USNO Data Set

\begin{tabular}{llcccc}
\hline \hline Filter & Obs. Date & $C_{0}$ & $C_{1}$ & $C_{2}$ & $\sigma_{\text {stds }}$ \\
\hline USNO V & 1994 Jul 8 & 4.938 & 0.000 & 0.161 & 0.008 \\
& 1995 Jun 24 & 4.885 & 0.010 & 0.170 & 0.015 \\
& 1995 Jun 25 & 4.885 & 0.010 & 0.177 & 0.014 \\
& 1998 Jun 23 & 3.720 & 0.014 & 0.126 & 0.016 \\
USNO I & 1994 Jul 8 & 5.480 & 0.029 & 0.062 & 0.008 \\
& 1995 Jun 24 & 5.390 & 0.039 & 0.076 & 0.020 \\
& 1995 Jun 25 & 5.390 & 0.039 & 0.079 & 0.019 \\
& 1998 Jun 23 & 4.408 & 0.024 & 0.054 & 0.010 \\
\hline
\end{tabular}

image. Therefore, potential variable stars were examined by eye to decide on real versus spurious variables. Table 2 also lists those stars that $\mathrm{B} \& \mathrm{~S}$ originally marked as variable candidates but which were found not to be variable in our survey. The instrumental magnitudes were shifted onto a common system, iteratively rejecting variable stars, using a method similar to that described by Honeycutt (1992).

Finally the USNO instrumental magnitudes were transformed to standard Johnson $V$ and Cousins $I$ magnitudes as follows. On three photometric nights when Draco images were taken in all quadrants, Landolt (1992) standards were also observed and used to determine transformation coefficients of the form

$$
V=v+C_{0}+C_{1} *(V-I)+C_{2} * \text { air mass }
$$

for $V$, with a similar form for $I$. On one additional photometric night, using a different Tektronix $1024 \times 1024$ CCD, images were taken centered on Draco, together with Landolt standards. Color coefficients were small, typically 0.01 and 0.03 in $V$ and $I$, respectively. These coefficients are presented in Table 3 for both $V$ and $I$ bands and per photometric night. Three nights were used to determine mean $V$ and $I$ standard magnitudes for a subset of bright (16-18 mag) nonvariable stars in the Draco images. The transformation of instrumental magnitudes (after shifting onto the common system) to standard magnitudes for this subset of bright stars then was applied to all stars. A comparison of the resulting standard magnitudes for nonvariable stars with Stetson's Draco calibration region ${ }^{6}$ shows good agreement.

The resulting errors in photometry for a single observation are estimated to be $0.01 \mathrm{mag}$ from calibration uncertainties, 0.02 mag from image distortion in the CCD corners, and photon noise that increases from $0.01 \mathrm{mag}$ at $V=18 \mathrm{mag}$ to $0.03 \mathrm{mag}$ at $V=20$ and to $0.05 \mathrm{mag}$ at $V=21 \mathrm{mag}$. After combining
Table 4

Transformation Coefficients for WIRO Data Set ${ }^{\mathrm{a}}$

\begin{tabular}{lcc}
\hline \hline Field & $\alpha_{V}$ & $\alpha_{I}$ \\
\hline WIRO Field 1 & $-3.453 \pm 0.002$ & $-1.734 \pm 0.003$ \\
WIRO Field 2 & $-3.421 \pm 0.002$ & $-1.715 \pm 0.002$ \\
WIRO Field 3 & $-3.426 \pm 0.002$ & $-1.769 \pm 0.003$
\end{tabular}

Note. ${ }^{\text {a }}$ The $\beta$ coefficient is $0.081 \pm 0.01$ and $\gamma=-0.09 \pm 0.01$ in Equation (2).

frames, the errors in the mean magnitudes of nonvariable stars at the level of the $\mathrm{HB}(V=20 \mathrm{mag})$ are estimated to be $0.03 \mathrm{mag}$ in $V, 0.03 \mathrm{mag}$ in $I$, and $0.04 \mathrm{mag}$ in $V-I$. The errors in the mean magnitudes of variable stars are generally larger.

\subsection{WIRO Observations}

The USNO data set was combined with data obtained at WIRO during the summer quarter observing season of 1993 and 1994. An RCA $337 \times 527$ pixel CCD camera was used, which had a 1'.2 pixel $^{-1}$ plate scale. The field of view was much smaller compared with the USNO data set. The WIRO fields were $6.4 \times 10^{\prime} .4$ and overlapped with three quadrants of the USNO fields. One WIRO field is roughly $13 \%$ of one USNO field. Figure 1 shows where the WIRO fields are in relation to the USNO fields. The data were obtained with Johnson $V$ and Cousins $I$ filters. From WIRO, a maximum of $28 \mathrm{~V}$ - and $18 I$-band images supplemented the USNO data. All available data from WIRO were used for light curve and period analysis of the variable stars. This brings a maximum of $69 \mathrm{~V}$ and $38 \mathrm{I}$ images for stars found in USNO and WIRO fields.

The WIRO observations were placed on a standard system by using secondary standards from the USNO analysis. A total of 45 stars were used for the calibration, and the dominant source of uncertainty is from the original calibration done with the USNO data set for each bandpass (see Section 2.1). Equations (2) and (3) are the transformation equations for the WIRO data set. The coefficients $\alpha_{V}$ and $\alpha_{I}$ were field dependent and were determined from a weighted mean of differences. The coefficients $\beta$ and $\gamma$ were obtained from a linear least-squares fit between $\left(V-v_{0}\right)$ and $(V-I)$. The standard $V$ magnitude was found through an iterative process, incorporating the standard I magnitude of that star. The values of the transformation coefficients for the WIRO data set are given in Table 4.

$$
\begin{gathered}
V=v_{0}-\alpha_{V}+\beta\left(v_{0}-I\right)+\gamma \\
I=i_{0}-\alpha_{I} .
\end{gathered}
$$


Photometry was performed on the WIRO data using Stetson's DAOPHOT II and ALLFRAME stand-alone packages (Stetson 1987; Stetson 1994).

\subsection{Variable Star Identifications}

We have kept the original numbering system of B\&S for the first 203 variable stars plus number 204 assigned by Zinn \& Searle (1976). All new variable star identifications, as well as the new long-period variable stars and the QSOs, are an extension of B\&S's system, but organized by right ascension going east to west. Our new star identification, therefore, begins from V205 through V333.

Stars with high dispersion or high chi-square for their magnitude were considered to be potential variables and inspected further. For the USNO data set, we used a plot of chi-squared versus magnitude to identify potential variables. We did not use the Welch \& Stetson variability index (Welch \& Stetson 1993) because it is defined to make use of pairs of images taken at nearly the same time, and the USNO data generally included unpaired images in each quadrant each night. Image differencing might in retrospect be useful as an additional tool; however, it is more advantageous in fields more crowded than Draco.

For the WIRO data set, the variable stars were selected by using a simple variability index which compared the external to internal uncertainties of the observations. Our results were then compared with the B\&S catalog and we identified the known variable stars. New variables were found and classified by their color, period, and location in the color-magnitude diagram (CMD). Due to the overlap of the WIRO fields with the USNO fields, the variable stars found were checked and confirmed between the two data sets.

A total of 270 RRL stars, 9 ACs, 12 semi-irregular or carbon stars, and 2 eclipsing binaries were discovered in this survey. We were able to recover all of the original $B \& S$ variable stars, and re-classified seven stars. We discuss the variable stars of Draco in more detail in Section 4.

\subsection{Comparison with Bonanos et.al. (2004)}

As discussed in Section 2.1, our survey of Draco is nearly four times larger in area and includes twice the number of variable stars than that found in the Bonanos et al. (2004) survey. Because of a match-up error in preparing their tables, the periods, magnitudes, and the identifications of 48 stars do not match the right ascension/declination star names in Bonanos' Tables 1 and 2. We have used corrected versions of the tables, provided by A. Bonanos, to make the comparison here. We independently recovered 130 stars that had been found in both the original B\&S and Bonanos et al. surveys. Those stars with the B04 designation in our Table 5 have been identified in Bonanos et al. They also identified 17 new RRL stars and one new eclipsing variable; we independently recover all 18 of these stars and make the same classifications, although we find one star (V289) to be an RRd that Bonanos et al. classified as RRab. For this star, we were able to find a period ( $P=0.6607$ days) close to the Bonanos et al. period, but it produced a noisy light curve with our data. Our solution produces a tighter light curve for our photometry. Bonanos et al. identified nine red variables with small amplitudes near the tip of the giant branch. We find that four of them vary, and find that the other five do not vary significantly in our data, so we omit them from our tables. Finally, the SX Phe star that Bonanos et al. found was too faint for our survey and was not included in our analysis. For
Table 5

Properties of Draco RRL Stars

\begin{tabular}{|c|c|c|c|c|c|c|c|}
\hline (1) & $\begin{array}{c}\text { R.A. } \\
(2000.0) \\
(2)\end{array}$ & $\begin{array}{c}\text { Decl. } \\
(2000.0) \\
(3)\end{array}$ & $\begin{array}{c}\text { Period } \\
\text { (days) } \\
(4)\end{array}$ & Amplitude & $\langle V\rangle^{\mathrm{a}}$ & $\langle I\rangle^{\mathrm{a}}$ & Type $^{b}$ \\
\hline 1 & $17: 20: 13.59$ & $58: 05: 24.2$ & 0.39363 & 0.48 & 20.18 & 19.60 & $\mathrm{c} ; \mathrm{B} 1 ?$ \\
\hline 2 & $17: 19: 42.54$ & $58: 03: 26.8$ & 0.59259 & 0.80 & 19.96 & 19.41 & $a b$ \\
\hline 3 & $17: 20: 14.88$ & $58: 01: 46.8$ & 0.64876 & 0.78 & 20.00 & 19.39 & $\mathrm{ab} ; \mathrm{B} 04$ \\
\hline 4 & $17: 20: 29.95$ & $58: 00: 57.7$ & 0.62625 & 0.61 & 20.18 & 19.63 & $\mathrm{ab} ; \mathrm{B} 04$ \\
\hline 6 & $17: 20: 18.95$ & 58:00:37.9 & 0.69485 & 0.75 & 19.94 & 19.34 & $\mathrm{ab} ; \mathrm{B} 04$ \\
\hline 7 & 17:20:09.56 & $57: 59: 57.4$ & 0.61896 & 0.62 & 20.19 & 19.57 & $\mathrm{ab} ; \mathrm{B} 04$ \\
\hline 8 & $17: 20: 15.23$ & $57: 59: 17.3$ & 0.56957 & 0.92 & 20.10 & 19.55 & $\mathrm{ab} ; \mathrm{B} 04$ \\
\hline 9 & 17:19:35.72 & $57: 58: 32.2$ & 0.68418 & 0.50 & 20.02 & 19.39 & $\mathrm{ab} ; \mathrm{B} 04$ \\
\hline 11 & $17: 20: 41.93$ & $57: 58: 27.4$ & & 0.48 & 20.08 & 19.52 & d;B04 \\
\hline 12 & $17: 20: 41.86$ & $57: 57: 50.0$ & 0.57638 & 0.76 & 20.17 & 19.61 & $\mathrm{ab} ; \mathrm{B} 04$ \\
\hline
\end{tabular}

Notes.

${ }^{\text {a }}$ Intensity-weighted magnitudes.

${ }^{\mathrm{b}}$ Bl: Blazhko effect.

References. B04: Bonanos et al. (2004).

(This table is available in its entirety in machine-readable and Virtual Observatory (VO) forms in the online journal. A portion is shown here for guidance regarding its form and content.)

most RRL stars, we find excellent agreement with the periods and RRab/RRc classifications with Bonanos et al. The typical difference between our periods and those of Bonanos et al. for the RRL stars is 0.00002 days. For a few stars, we find a different alias period. The greater number of nights covered by our observations usually make alias problems less important in our analysis, so we prefer our period solutions.

\section{DATA ANALYSIS}

Once the data sets from USNO and WIRO were independently reduced, the data were combined. This increased the number of epochs for 103 variable stars. Using our combined data sets, we present a robust CMD of the Draco $\mathrm{dSph}$ galaxy down to a limiting magnitude of $V=21 \mathrm{mag}$ in Figure 2. In this updated CMD, we have identified pulsating and eclipsing stars as well as background QSOs in the Draco field. Our census has yielded 279 stars that are either of the RRL or Cepheid type of pulsating variable star. We have found 12 variable stars which were not RRL, ACs, or eclipsing stars, but belong to other typeseither slow, semi-regular, red, or other objects. There appear to be 30 background QSOs found in our coverage of the Draco galaxy. The rest of the stars plotted in Figure 2 are nonvariable (approximately 4700 stars). There is also contamination of field stars from the Milky Way, and thus a likelihood of field RRL in our survey. We address this possibility in Section 4.1. Figure 3 is a close up view of the HB region of the CMD. Here, we identify the individual RRL Bailey types, as well as the nonvariable stars. We note that there is a large scatter of nearly 0.4 mag for the RRL.

The subsequent analysis was done in four steps: (1) period searching, (2) amplitude and mean magnitude calculation, (3) Fourier decomposition of the light curves, and (4) deriving distances from the RRL population. The Fourier decomposition work is discussed in detail in Section 4.2.

For the full data set, we anticipated minimizing any period alias solutions, specifically any yearly aliases. Our primary period searching method was the date compensated discrete Fourier transform (DCDFT) program (Ferraz-Mello 1981; Foster 1995). This program was particularly useful for data sets 


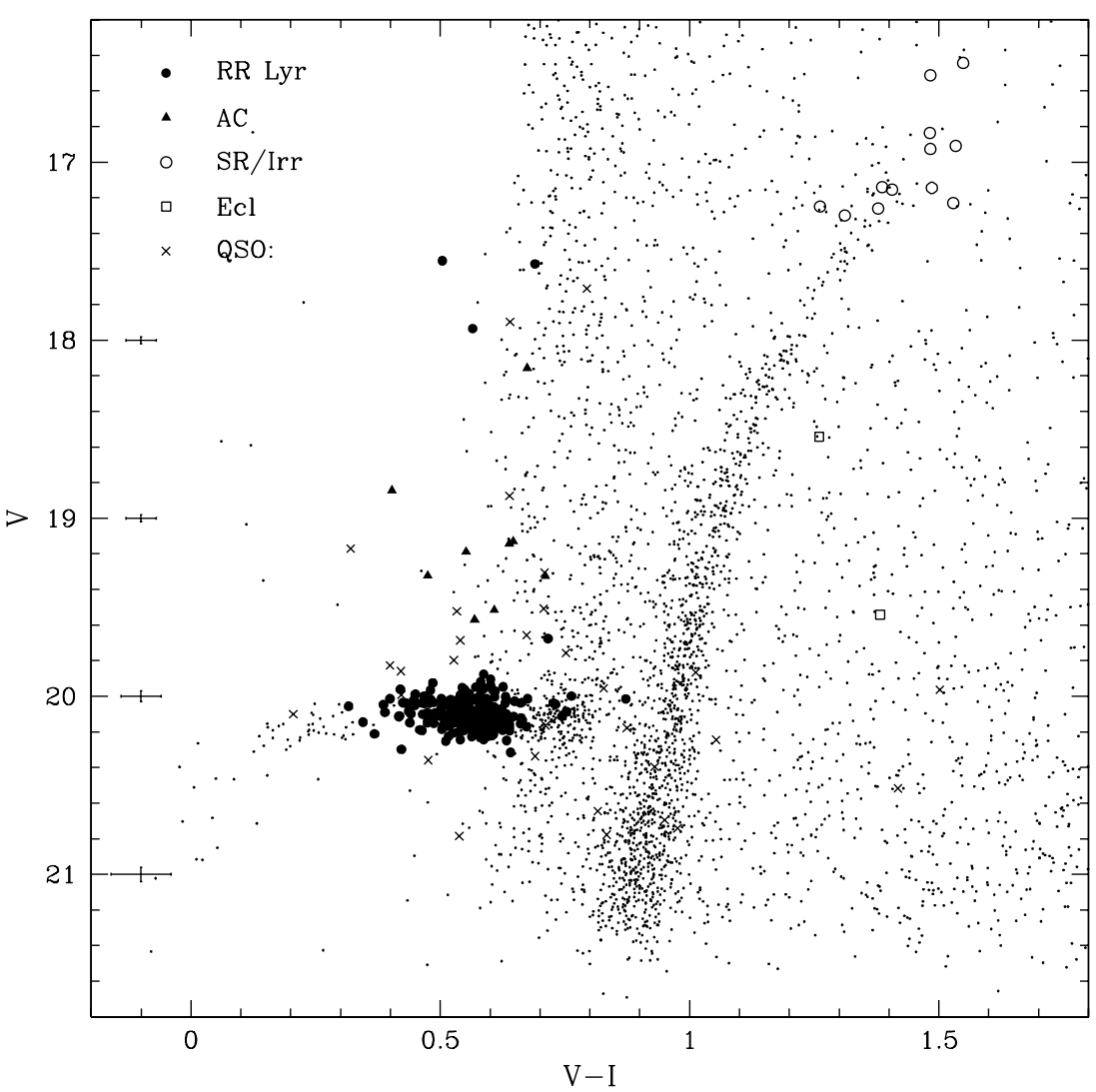

Figure 2. CMD of the Draco dSph galaxy. Variable stars (RRL, Cepheids, eclipsing binaries, and semi-regular) are identified in the figure. Background QSOs are included in this diagram. Representative error bars for nonvariable stars are shown at the left edge of the figure.

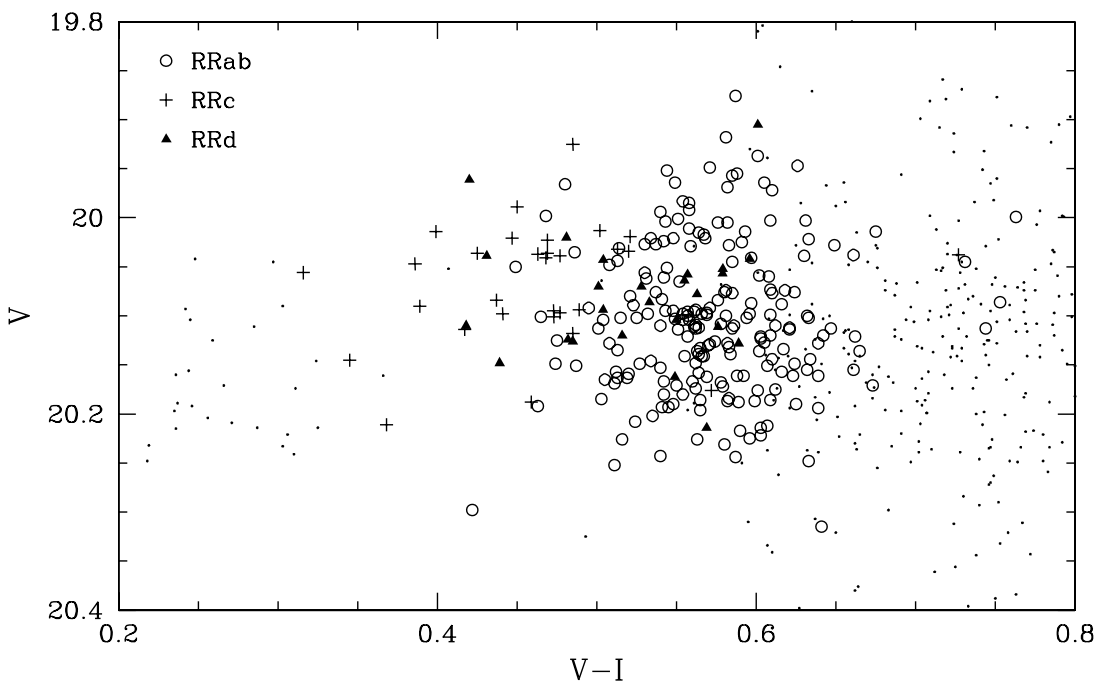

Figure 3. Closer view of the HB. RRab stars are the open circles, RRc stars are the plus signs, and the RRd stars are the filled triangles.

that have a patchy distribution of data points (i.e., the observations were more or less annual). The actual DCDFT program is part of the CLEANest program (Foster 1995). An updated version of this program is available through Peranso. ${ }^{7}$ As a check for the period solutions, the IRAF version of the phase dispersion minimization statistic (PDM) (Stellingwerf 1978) was used, as well as the Supersmoother routine (Reimann 1994). Overall, our periods are good to about 0.00001-0.00003 days. To obtain the amplitudes of the $V$ and $I$ variable star data, we use a spline fit to the phased light curve.

\footnotetext{
7 www.peranso.com
}

\section{VARIABLE STAR CENSUS}

\subsection{RR Lyrae Stars}

Figure 4 shows the phased $V$ and $I$ light curves. The $V$ light curves have our best spline fit included to aid the eye. Fourier series fits to our light curves were not used because they often give biased results at rapidly changing phases (rising and maximum light) if few data points are available to constrain the fit. With typically $40 \mathrm{~V}$ observations, some stars in our data have few points at these phases. Table 5 lists the RRL positions (R.A. and decl. J2000.0), the period solutions (Column 4), the $V$ amplitude (Column 5), the intensity-weighted mean 

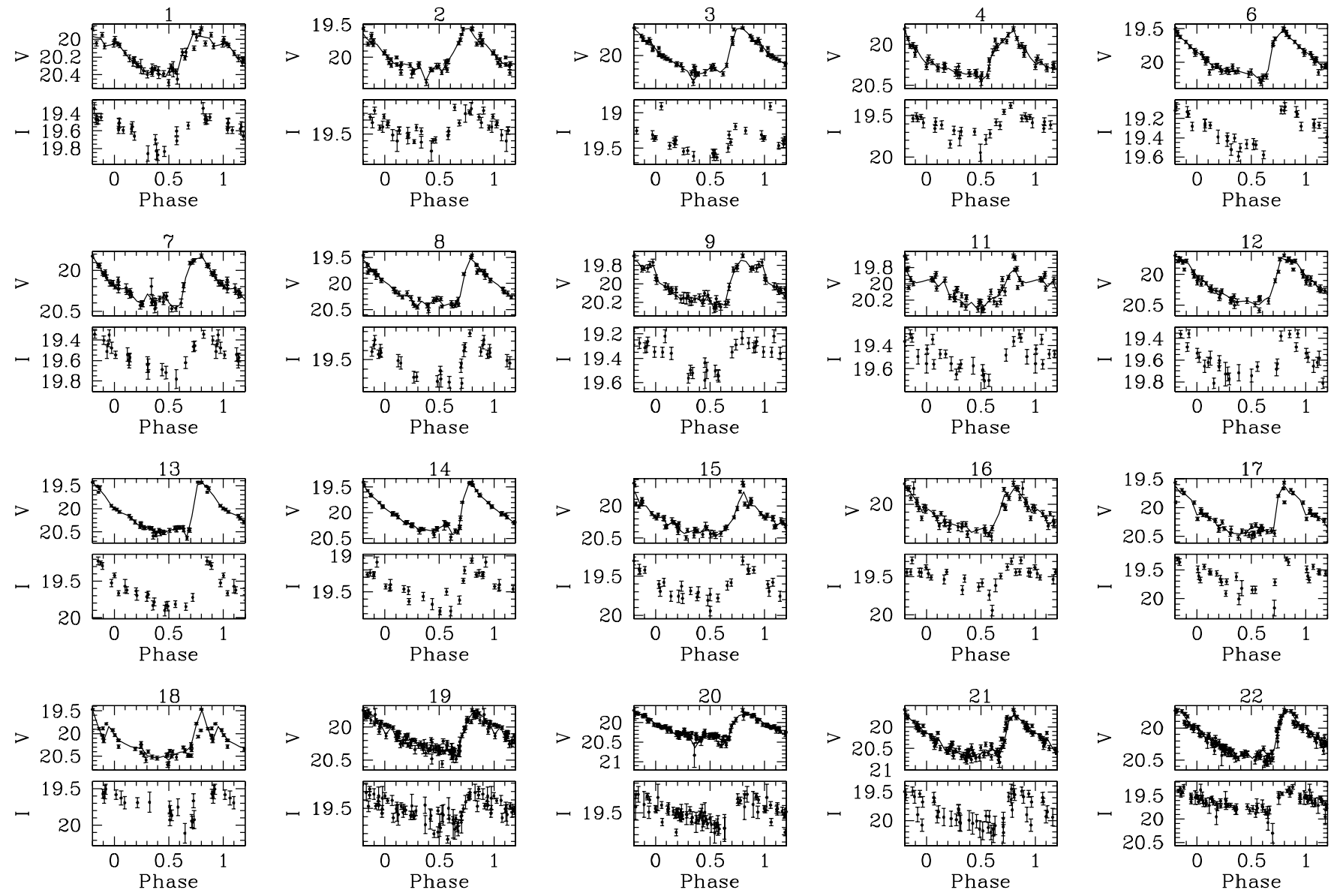

Figure 4. Phased light curves of the Draco RRL population. We present a sample of these light curves here.

(An extended version of this figure is available in the online journal.)

magnitudes in $V$ and $I$ (Columns 6 and 7, respectively), and the type of RRL with additional notes (Column 8). We find in our survey 270 RRL stars, of which 214 are RRab, 30 RRc, and 26 RRd stars. Of these, 81 are new RRL compared to the B\&S study. Including these new RRL stars, we find the average period of the RRab stars to be $\left\langle P_{a b}\right\rangle=0.615 \pm 0.003$ days and for the RRc stars an average period $\left\langle P_{c}\right\rangle=0.375 \pm 0.006$ days. In Figure 5, we show the period distribution of the RRL stars. The average period for the RRd stars is $\left\langle P_{d}\right\rangle=0.407 \pm 0.002$ days. As originally noted by $B \& S$, the mean period of the RRab stars is the Oosterhoff intermediate. The Oosterhoff properties of the Draco dwarf system are discussed in detail in Section 5.

Foreground RRL have been found in our survey. Using the surface density for RRL in the SA57 field (Kinman et al. 1994), and assuming a halo space density of $R^{-3.5}$, we calculated the volume and RRL per magnitude along our line of sight. From the calculation, we expected 0.9 field RRL in the line of sight, but in actuality we find three field RRL (V327, V321, and V276). One of these stars (V327) was previously discovered by Wehlau et al. (1986). The distribution of stars per magnitude peaked around $V=17 \mathrm{mag}$, thus we should see field RRL around this magnitude. The three field RRL are flagged in the main RRL properties table, Table 5.

\subsubsection{Double-Mode RR Lyrae Stars}

Goranskij (1982) used the photometry of B\&S to identify three RRL stars in Draco that were pulsating simultaneously in the first-overtone and fundamental radial modes. Also using the B\&S observations, Nemec (1985a) identified seven more of these stars (RRd variables in Nemec's nomenclature, or RR01 stars in the nomenclature of Clement et al. 2001). Bonanos et al. (2004) redetermined periods for six of the RRd stars found by Nemec (1985a).

We carried out a search for double-mode behavior among the RRL stars that had light curves that did not seem to be adequately described by a single period. Using the CLEANest routine (Foster 1995) to prewhiten the $V$-band observations, we removed the primary frequency and its first four harmonics. A search was then undertaken for evidence of a significant secondary period. If a secondary period seemed possible, the CLEANest routine was used to simultaneously fit the primary and secondary periods and their first four harmonics. Although higher-order harmonics and cross-frequency terms have been detected in the light curves of double-mode RRL stars, the current set of observations is not sufficient to identify them. For suspected RRd stars, results from the CLEANest routine were verified using the Period04 program (Lenz \& Breger 2005).

By this means we found all ten of the RRd stars identified by Goranskij (1982) and Nemec (1985a). In addition, we have identified 16 probable RRd variables, giving a total of 26 . The first-overtone mode was the dominant mode in each case. First-overtone mode periods, fundamental mode periods, and period ratios for probable RRd stars are shown in Table 6. The listed uncertainties are the formal errors given by the CLEANest program. Results for stars with asterisks are more 


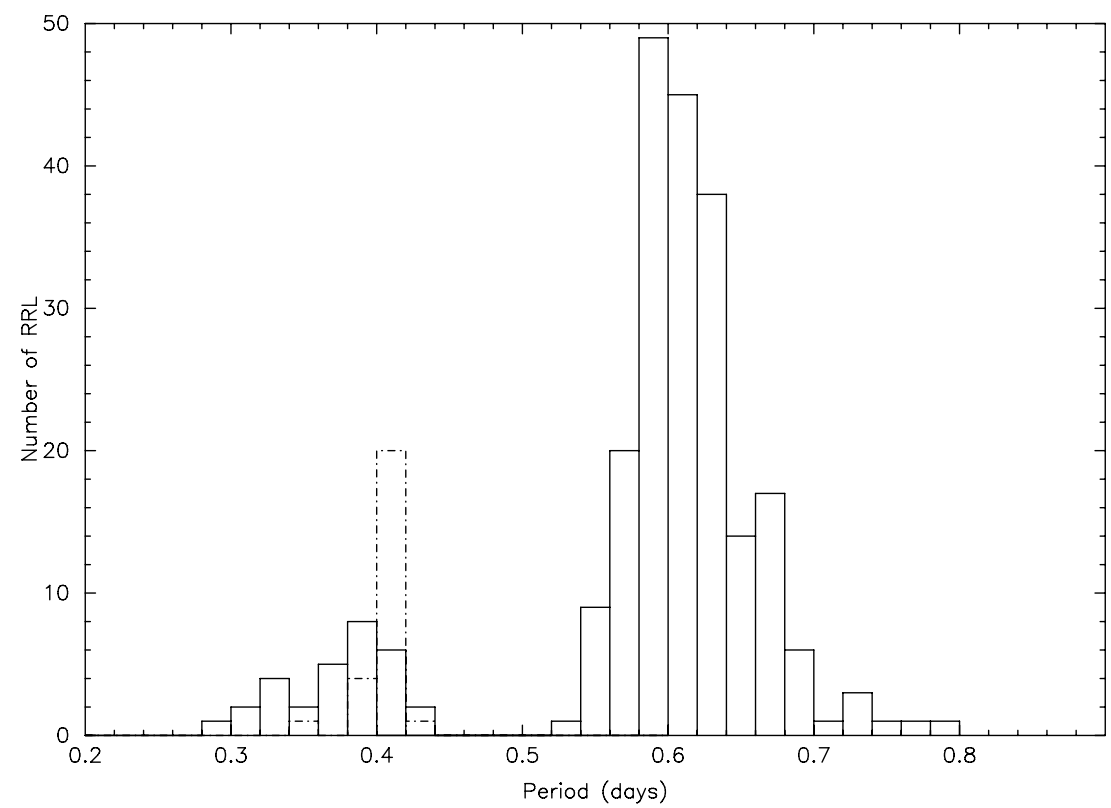

Figure 5. Period distribution of all Draco RRL stars. The dash-dotted histogram is of the double-mode RRLs. Average periods for each Bailey type of RRL: $\left\langle P_{a b}\right\rangle=0.615$ days, $\left\langle P_{c}\right\rangle=0.375$ days, and $\left\langle P_{d}\right\rangle=0.407$ days.

Table 6

Properties of the Draco RRd Stars

\begin{tabular}{rcccccl}
\hline \hline ID & $P_{1}$ & Error & $P_{0}$ & Error & $P_{1} / P_{0}$ & Error $^{\mathrm{a}}$ \\
\hline 11 & 0.41100 & 0.00002 & 0.55114 & 0.00010 & 0.7457 & 0.0002 \\
72 & 0.40711 & 0.00002 & 0.54599 & 0.00006 & 0.7456 & 0.0001 \\
83 & 0.40075 & 0.00002 & 0.53720 & 0.00006 & 0.7460 & 0.0001 \\
112 & 0.42844 & 0.00002 & 0.57446 & 0.00005 & 0.7458 & 0.0001 \\
131 & 0.40626 & 0.00002 & 0.54424 & 0.00006 & 0.7465 & 0.0001 \\
138 & 0.40773 & 0.00003 & 0.54601 & 0.00012 & 0.7467 & 0.0002 \\
143 & 0.40317 & 0.00004 & 0.54042 & 0.0003 & 0.7460 & $0.0006^{*}$ \\
155 & 0.41393 & 0.00003 & 0.55476 & 0.00009 & 0.7461 & $0.0002^{*}$ \\
156 & 0.40868 & 0.00002 & 0.54778 & 0.00006 & 0.7461 & 0.0001 \\
165 & 0.35798 & 0.00002 & 0.48064 & 0.00004 & 0.7448 & 0.0001 \\
169 & 0.40316 & 0.00003 & 0.54059 & 0.00008 & 0.7458 & 0.0002 \\
190 & 0.39652 & 0.00001 & 0.53080 & 0.00006 & 0.7470 & $0.0001^{*}$ \\
217 & 0.41166 & 0.00003 & 0.55149 & 0.00014 & 0.7465 & $0.0002^{*}$ \\
221 & 0.40788 & 0.00003 & 0.54671 & 0.00008 & 0.7461 & 0.0002 \\
228 & 0.41606 & 0.00003 & 0.55784 & 0.00010 & 0.7458 & 0.0002 \\
232 & 0.41081 & 0.00001 & 0.55017 & 0.00007 & 0.7467 & $0.0001^{*}$ \\
235 & 0.39954 & 0.00003 & 0.53560 & 0.00011 & 0.7460 & 0.0002 \\
245 & 0.41105 & 0.00001 & 0.55029 & 0.00010 & 0.7470 & $0.0002^{*}$ \\
247 & 0.41760 & 0.00002 & 0.55946 & 0.00006 & 0.7464 & 0.0001 \\
248 & 0.41828 & 0.00002 & 0.56055 & 0.00005 & 0.7462 & 0.0001 \\
250 & 0.40491 & 0.00002 & 0.54218 & 0.00014 & 0.7468 & $0.0003^{*}$ \\
289 & 0.39743 & 0.00002 & 0.53258 & 0.00008 & 0.7462 & 0.0002 \\
294 & 0.39998 & 0.00002 & 0.53622 & 0.00007 & 0.7459 & $0.0001^{*}$ \\
301 & 0.41286 & 0.00002 & 0.55306 & 0.00007 & 0.7465 & 0.0002 \\
306 & 0.39824 & 0.00002 & 0.53323 & 0.00009 & 0.7468 & 0.0002 \\
318 & 0.40264 & 0.00004 & 0.53995 & 0.00007 & 0.7457 & $0.0002^{*}$ \\
& & & & & &
\end{tabular}

Note. ${ }^{\text {a }}$ Stars with an asterisk $(*)$ denote some uncertainty with the period solutions due to aliasing.

uncertain, usually because of the possibility of a period alias for the fundamental mode period. Deconvolved first-overtone and fundamental-mode period light curves for the RRd stars are shown in Figure 6.

In plotting the Petersen diagram (Petersen 1973) of period ratio versus fundamental period, Nemec (1985a) discovered that V165 had a position in this diagram similar to those seen among
RRd stars in Oosterhoff type I globular clusters, but that all of the other stars had properties similar to those of RRd stars in Oosterhoff type II clusters. Figure 7 shows the Petersen diagram for all 26 probable RRd stars. RRd stars whose locations in this diagram are somewhat uncertain (the asterisked stars in Table 6) are plotted as open points. For comparison, the locations of RRd stars in the Oosterhoff type I globular cluster IC 4499 (Walker \& Nemec 1996) and the Oosterhoff type II globular clusters M15 and M68 (Nemec 1985b; Walker 1994) are also plotted. V165 still remains the only RRd star with properties similar to those of RRd stars in Oosterhoff type I clusters.

Figure 8 plots the luminosity-weighted mean $V$ magnitude against the primary period for all of the Draco RRL stars. V165, the sole Oosterhoff type I RRd star, is also the faintest RRd star. This is at least qualitatively consistent with other findings that RRL stars in Oosterhoff type I clusters are less luminous than those in Oosterhoff type II systems (e.g., Sandage 1958; Sandage et al. 1981).

\subsubsection{Blazhko Effect}

The Blazhko effect is a second-order modulation most evident in the shape of the RRL light curve. The maximum light phase can be depressed by the Blazhko effect. This effect is also periodic - on the order of tens to hundreds of days. What causes the Blazhko effect is not clearly known, but there are several proposed explanations (see Kolenberg et al. 2006; Stothers 2006).

We do not have enough observations to determine Blazhko periods for those RRL stars in our sample that show the Blazhko effect. We can, however, identify as Blazhko-effect candidates those RRL stars that have unusually large scatter in their light curves and which do not seem to be RRd stars. We list these Blazhko candidates in Table 5 by noting "Bl" in the last column. Stars V26, V33, V34, V35, V37, V39, V41, V68, V75, V96, V123, V129, V147, V150, V160, V184, and V196 have already been identified as possible Blazhko variables by Nemec (1985a) and Bonanos et al. (2004). The mean period of the Blazhko effect candidates among the RRab stars is $\left\langle P_{\mathrm{Bl}}\right\rangle=0.603 \pm 0.006$ days. 

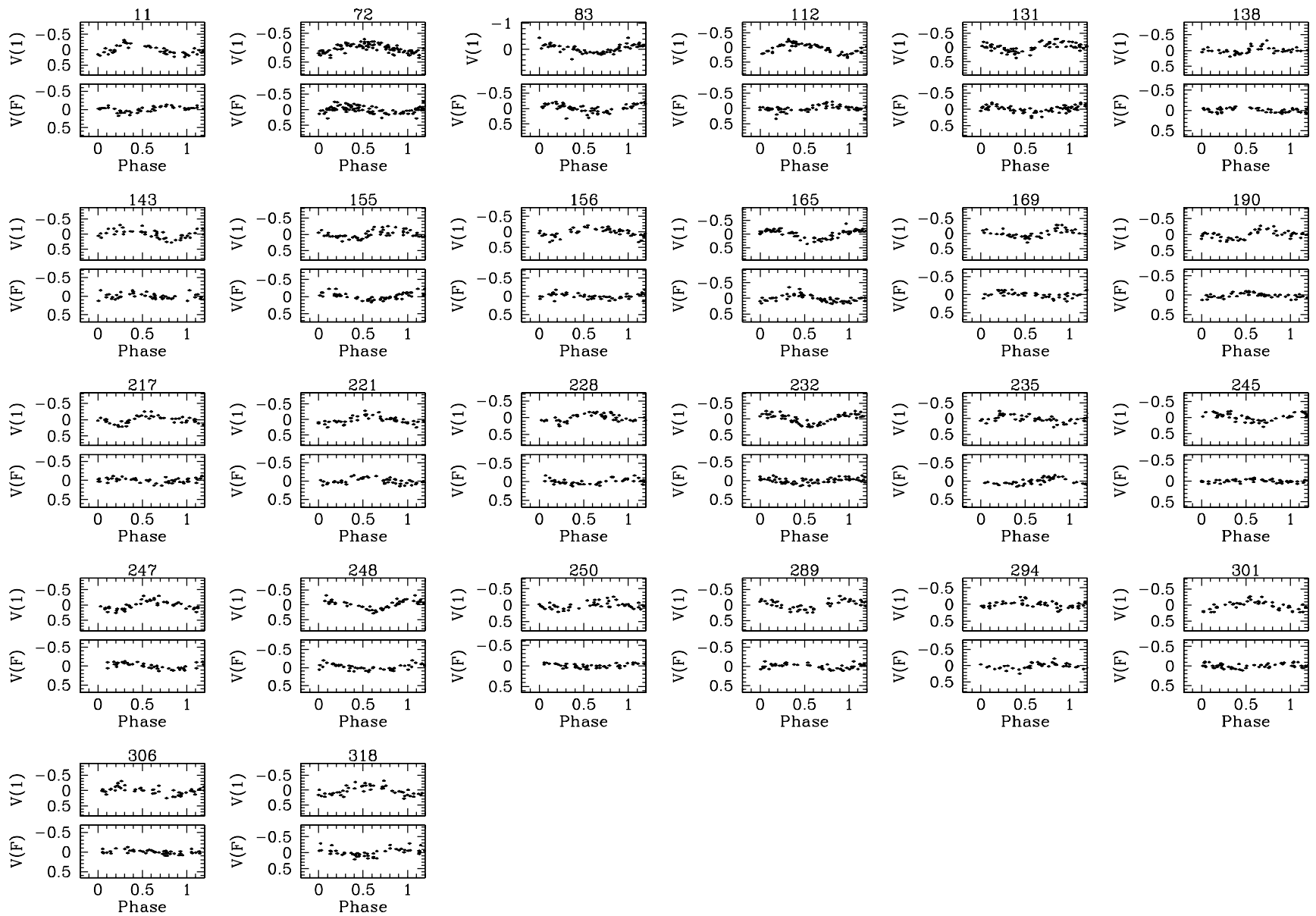

Figure 6. Deconvolved light curves of the Draco RRd stars. The upper plots are the first-overtone pulsation while the lower are of the fundamental mode.

\subsection{Fourier Decomposition}

The Fourier decomposition of the light curves was done only on the $V$ data. Using Simon's MINFIT program (Simon 1979; Simon \& Teays 1982), a cosine series up to 8th order was fit to the light curves:

$$
m=A_{0}+\sum A_{i} \cos \left(i \omega\left(t-t_{0}\right)+\phi_{0}\right) \quad \text { where } \quad i=1,2 \ldots
$$

Once the amplitude $\left(A_{i}\right)$ and phase $\left(\phi_{i}\right)$ terms were obtained, the Fourier parameters, $R_{j i}$ and $\phi_{j i}$, were calculated up to the 4 th order.

We applied the Jurcsik \& Kovacs (1996) photometric metallicity relation using the Fourier decomposition parameter $\phi_{31}$ and the period (their Equation (3)). The Jurcsik \& Kovacs method works best when RRab light curves are fully sampled and where photometric uncertainties are relatively small. The light curves for individual RRab stars in our sample do not always meet these criteria. To test the quality of the RRab light curve for this method, a compatibility test called the $D_{M}$ deviation parameter is calculated. This deviation parameter is determined from a comparison of the observed and predicted Fourier parameters. An updated version of this test is provided in Kovacs \& Kanbur (1998). In order for a star to be a good candidate for the Jurcsik \& Kovacs method, the $D_{M}$ parameter criterion must be met. For our RRab sample, we chose $D_{M}<3.0$ (as recommended by Jurcsik \& Kovacs) and $D_{M}<5.0$ (as recommended by Clement \& Shelton 1999). Stars that have passed the criteria are listed in Table 7 with asterisks. Table 7 also lists the Fourier decomposition parameters and photometric metallicities of the Draco RRab stars. All photometric metallicities are on the metallicity scale of the Jurcsik \& Kovacs method (Jurcsik 1995).

The $[\mathrm{Fe} / \mathrm{H}]$ values derived from the Jurcsik \& Kovacs (1996) method may in this case be more useful in deriving a mean $[\mathrm{Fe} / \mathrm{H}]$ value for Draco than in the determination of metallicities for individual stars. It is quite likely that some of the outlying $[\mathrm{Fe} / \mathrm{H}]$ values in Table 7 , at both the high and low ends, do not really reflect the metallicities of the stars for which they are derived. The average $[\mathrm{Fe} / \mathrm{H}]$ for Draco, as determined by the photometric metallicities of the RRab stars, is $\langle[\mathrm{Fe} / \mathrm{H}]\rangle=$ $-2.19 \pm 0.03$ if we assume the stars are not undergoing the Blazhko effect (see Section 4.1.1) and have passed the $D_{M}<3.0$ criterion. For the case where $D_{M}<5.0$, and assuming no Blazhko effect, the average metallicity of Draco is $\langle[\mathrm{Fe} / \mathrm{H}]\rangle=-2.23 \pm 0.03$. Figure 9 shows the metallicity distribution of the RRab stars that have passed the $D_{M}<5.0$ criterion with respect to period.

Using Stromgren photometry, Faria et al. (2007) recently obtained a mean $[\mathrm{Fe} / \mathrm{H}]$ of -1.74 for Draco and field red giant branch (RGB) stars, with most stars falling within the limits $-2.0<[\mathrm{Fe} / \mathrm{H}]<-1.5$. This result is broadly consistent with the earlier results of Shetrone et al. (2001a) and Zinn (1978), although Shetrone et al. (2001a) did find one red giant star as metal poor as $[\mathrm{Fe} / \mathrm{H}]=-2.97$. Faria et al. (2007) calibrated their derived metallicities to the work of Hilker (2000), which analyzed the red giants of three globular clusters and spanned a metallicity range of -2.0 to $0.0 \mathrm{dex}$. Therefore, 


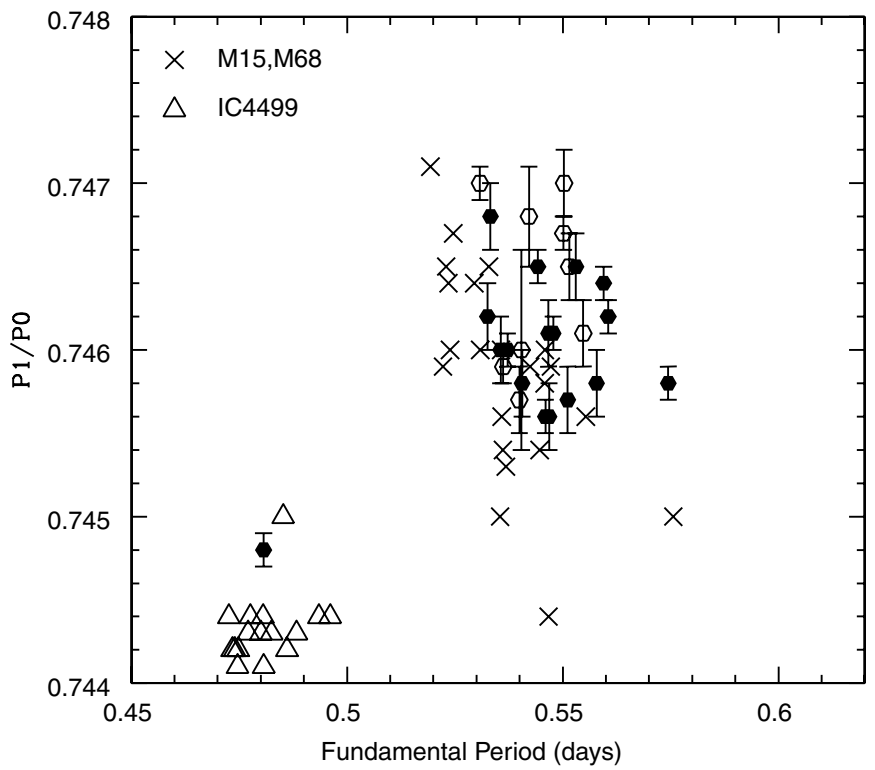

Figure 7. Petersen diagram of Draco RRd stars. The Draco RRd stars with uncertainties in the period are plotted as open circles. For comparison, RRd stars from the Oosterhoff type II clusters M15 (Nemec 1985b; Purdue et al. 1995) and M68 (Walker 1994) and Oosterhoff type I cluster IC 4499 (Walker \& Nemec 1996) are included.

we must be cautious when comparing out metallicity results with those of other studies since there are dependences to various metallicity calibrations. However, there is a suggestion that the average metallicity of the Draco RRab stars is lower than that of the Draco red giant stars. The reality of this difference in metallicity is uncertain due to the nature of the different calibration methods. If this difference is real, then presumably the red HB stars in Draco would have to be more metal rich on average than the RRab stars.

\subsection{RRL Distance for Draco}

Since RRL are excellent distance indicators, we calculate the distance to the Draco dwarf galaxy. We use the metalpoor $([\mathrm{Fe} / \mathrm{H}]<1.5)$ relation from Cacciari \& Clementini (2003) (their Equation (4)). As with the work of Bonanos et al. (2004), we use an $E(B-V)=0.027$ from the Schlegel et al. (1998) reddening maps and the corrections for the extinction as suggested by the work of Cardelli et al. (1989), thus, $A_{V}=0.091$. From our sample of RRL stars, the intensityweighted mean $V$ magnitude is $\langle V\rangle=20.10 \pm 0.04$ mag $\left(\sigma_{\mathrm{RMS}}=0.08\right)$. For this value, we omitted the magnitudes of the foreground RRL (V276, V321, and V327) and V176, since it is blended with a bright star. The uncertainties given for this mean magnitude account for the calibration errors, image distortion, and photon noise (see Section 2.1). The value of $\langle V(\mathrm{RR})\rangle=20.10 \pm 0.04 \mathrm{mag}$ in this paper is brighter than those of Bonanos et al. (2004): $\langle V(\mathrm{RR})\rangle=20.18 \pm 0.02 \mathrm{mag}$, those of Aparicio et al. (2001): $\langle V(\mathrm{HB})\rangle=20.2 \pm 0.1 \mathrm{mag}$, and those of Bellazzini et al. (2002): $\langle V(\mathrm{HB})\rangle=20.28 \pm 0.10 \mathrm{mag}$, with a $2 \sigma$ difference from the most precise value of Bonanos et al.

If we assume a metallicity for Draco from our Fourier decomposition analysis, $[\mathrm{Fe} / \mathrm{H}]=-2.19 \pm 0.03$, and using the Cacciari \& Clementini (2003) relation, our resultant absolute magnitude is $\left\langle M_{V}\right\rangle=0.43 \pm 0.13 \mathrm{mag}$. Therefore, using the present mean $V$ magnitude of the RRL stars and accounting for the extinction, we derive a dereddened distance modulus

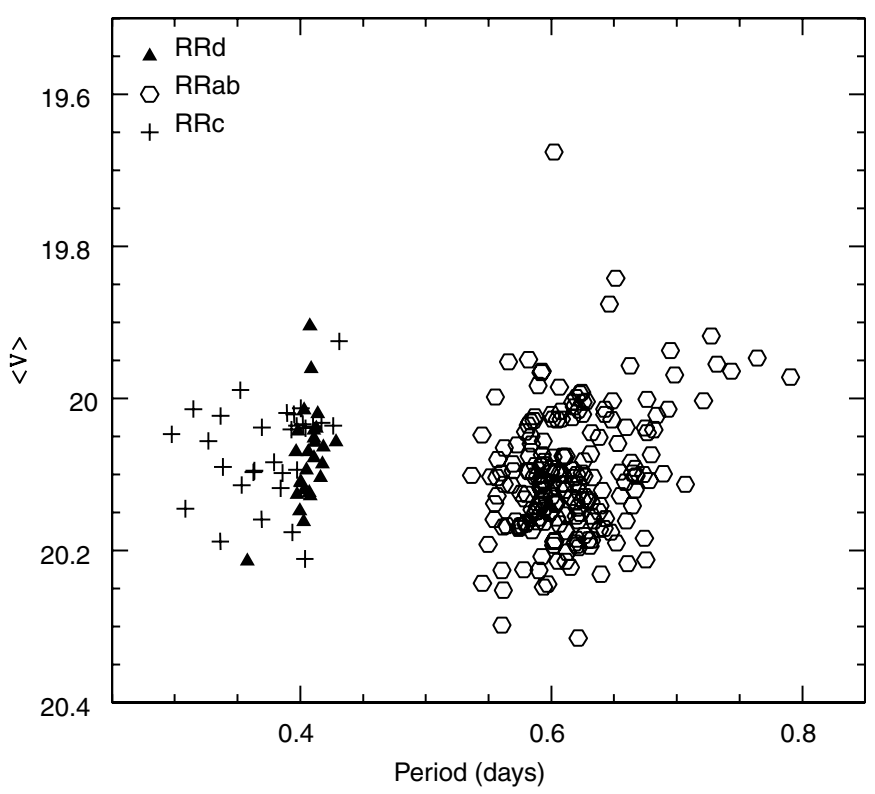

Figure 8. Intensity-weighted mean $V$ magnitude is plotted against period for all RRL stars in our study. The open circles are the RRab, plus signs are the RRc, and the filled triangles are the RRd stars.

to Draco of $\mu_{0}=19.58$, or $D=82.4 \pm 5.8 \mathrm{kpc}$. However, if we assume a different metallicity for Draco, our distance changes slightly. Shetrone et al. (2001a) obtained a mean metallicity of $[\mathrm{Fe} / \mathrm{H}]=-2.00 \pm 0.21$ from high-resolution spectroscopy of Draco red giants, whereas Faria et al. (2007) found $[\mathrm{Fe} / \mathrm{H}]=-1.74$. If we assume the metallicity values of -2.00 and -1.74 , and using the same Cacciari \& Clementini relation and the present RRL mean $V$ magnitude, the resultant distances are 81.2 and $79.8 \mathrm{kpc}$, respectively. Pritzl et al. (2002a) arrived at a distance to Draco independently of the AC stars (see Section 4.4). Their value is $\mu_{0}=19.49$ or $D=79.1 \mathrm{kpc}$, but using a reddening value of $E(B-V)=0.03$. Within our uncertainties, we agree with all these distance values from different Draco studies.

\subsection{Anomalous Cepheids}

In our study of the Draco dwarf galaxy, we increase the number of known ACs to nine. B\&S had identified what appeared to be five overly bright RRL stars in their original survey. Norris \& Zinn (1975), followed by Zinn \& Searle (1976), first classified these variables as AC stars (V134, V141, V157, V194, and V204). Nemec et al. (1988) re-identified the five stars in Draco as AC, based on a re-analysis of B\&S's photographic survey. These five AC stars were confirmed in our study. We have been able to add four new ACs (V31, V230, V282, and V312) to the census. Table 8 lists all the photometrically-derived parameters.

Of the new ACs, one star, V31, has been re-classified. Originally, it was identified by B\&S as an RRL variable star based on eye estimates only. However, it lies only $13^{\prime \prime}$ from a bright BD star. The $I$ and the $V-I$ colors are particularly uncertain because of scattered light from the nearby bright red star. Our CCD data show that it is significantly brighter than other Draco RRL stars, so we believe it is a new AC. The bright star is saturated in our data and contributes significant scattered light around V31. Nevertheless, after doing careful background subtraction, the estimated errors in our photometry are 0.1 in $V$ and 0.2 in $I$, leaving it 0.5 mag brighter than the HB. The other AC stars do not have companions visible in 
Table 7

Fourier Decomposition Parameters for RRab Stars

\begin{tabular}{|c|c|c|c|c|c|c|c|c|c|c|c|}
\hline ID & $A_{0}$ & $R_{21}$ & $R_{31}$ & $R_{41}$ & $\phi_{21}$ & $\phi_{31}$ & $\phi_{41}$ & $\sigma_{\phi_{31}}$ & {$[\mathrm{Fe} / \mathrm{H}]$} & $\sigma_{[\mathrm{Fe} / \mathrm{H}]}$ & $D_{M}$ Pass? $^{\mathrm{a}}$ \\
\hline 2 & 20.9797 & 0.3221 & 0.0858 & 0.1693 & 3.5756 & 1.403 & 5.449 & 0.553 & -2.43 & 0.75 & \\
\hline 3 & 21.0260 & 0.3453 & 0.2780 & 0.2168 & 3.9108 & 1.569 & 6.036 & 0.180 & -2.51 & 0.25 & $*$ \\
\hline 4 & 21.1916 & 0.4781 & 0.1509 & 0.0581 & 3.8671 & 1.302 & 1.641 & 0.356 & -2.76 & 0.48 & \\
\hline 6 & 20.9611 & 0.5028 & 0.2893 & 0.1936 & 3.8149 & 2.042 & 6.092 & 0.134 & -2.11 & 0.18 & \\
\hline 7 & 21.2041 & 0.4867 & 0.3420 & 0.0415 & 3.7750 & 1.915 & 5.866 & 0.179 & -1.86 & 0.24 & \\
\hline 8 & 21.1371 & 0.5084 & 0.3504 & 0.2033 & 3.8696 & 1.671 & 6.105 & 0.128 & -1.92 & 0.17 & $*$ \\
\hline 9 & 21.0360 & 0.4902 & 0.2396 & 0.1688 & 3.7757 & 1.799 & 0.785 & 0.222 & -2.39 & 0.30 & \\
\hline 12 & 21.2055 & 0.4099 & 0.2215 & 0.1029 & 3.8709 & 1.743 & 5.930 & 0.238 & -1.86 & 0.32 & $*$ \\
\hline 13 & 21.1566 & 0.4181 & 0.2999 & 0.1742 & 3.8595 & 1.408 & 5.677 & 0.108 & -2.10 & 0.15 & $*$ \\
\hline 14 & 21.0536 & 0.3410 & 0.3305 & 0.3288 & 3.8314 & 1.857 & 5.995 & 0.173 & -1.93 & 0.23 & $*$ \\
\hline 15 & 21.2359 & 0.4504 & 0.2656 & 0.0374 & 3.5087 & 1.533 & 5.400 & 0.278 & -2.16 & 0.38 & $*$ \\
\hline 16 & 21.1378 & 0.3367 & 0.1520 & 0.0652 & 4.0760 & 1.823 & 4.995 & 0.453 & -2.02 & 0.61 & \\
\hline 17 & 21.1852 & 0.3700 & 0.2613 & 0.0471 & 3.5913 & 1.052 & 6.038 & 0.272 & -2.96 & 0.37 & \\
\hline 19 & 21.1497 & 0.4018 & 0.2557 & 0.1974 & 4.1254 & 2.255 & 0.236 & 0.186 & -1.45 & 0.25 & \\
\hline 20 & 21.1713 & 0.4051 & 0.3398 & 0.2134 & 3.8324 & 1.714 & 0.185 & 0.159 & -2.15 & 0.22 & \\
\hline 21 & 21.3392 & 0.3626 & 0.2600 & 0.2263 & 3.7277 & 1.337 & 5.622 & 0.199 & -2.34 & 0.27 & $*$ \\
\hline 22 & 21.1894 & 0.3796 & 0.3104 & 0.1628 & 4.1072 & 1.950 & 0.004 & 0.286 & -1.59 & 0.39 & \\
\hline 23 & 21.1370 & 0.5135 & 0.2314 & 0.2189 & 3.7667 & 1.487 & 5.489 & 0.425 & -2.45 & 0.57 & \\
\hline 24 & 21.1933 & 0.3830 & 0.2605 & 0.1930 & 4.0358 & 1.462 & 0.398 & 0.177 & -2.57 & 0.24 & \\
\hline 25 & 21.2862 & 0.3835 & 0.3411 & 0.3119 & 4.0705 & 1.710 & 5.930 & 0.136 & -1.82 & 0.18 & $*$ \\
\hline 27 & 20.9620 & 0.4201 & 0.3533 & 0.0944 & 3.9173 & 2.328 & 0.985 & 0.156 & -2.09 & 0.21 & \\
\hline 28 & 21.1103 & 0.4367 & 0.3305 & 0.1880 & 3.9909 & 1.712 & 6.234 & 0.156 & -2.18 & 0.21 & $*$ \\
\hline 29 & 21.0918 & 0.5195 & 0.6465 & 0.1672 & 3.4223 & 1.529 & 5.983 & 0.225 & -2.12 & 0.30 & \\
\hline 30 & 21.2023 & 0.3684 & 0.2356 & 0.1205 & 3.6366 & 2.060 & 0.933 & 0.183 & -1.71 & 0.25 & \\
\hline 32 & 21.0954 & 0.3225 & 0.3235 & 0.3422 & 3.9962 & 2.185 & 5.995 & 0.270 & -1.09 & 0.36 & \\
\hline 33 & 21.1307 & 0.3173 & 0.3863 & 0.1106 & 3.7809 & 1.359 & 6.199 & 0.155 & -2.59 & 0.21 & \\
\hline 34 & 21.2633 & 0.4045 & 0.2217 & 0.2377 & 4.2836 & 2.324 & 0.540 & 0.206 & -0.86 & 0.28 & \\
\hline 36 & 21.0509 & 0.3891 & 0.3177 & 0.2871 & 3.9562 & 1.524 & 5.890 & 0.100 & -2.44 & 0.14 & $*$ \\
\hline 37 & 21.2053 & 0.3731 & 0.2829 & 0.1995 & 3.6804 & 1.418 & 5.892 & 0.155 & -2.19 & 0.21 & $*$ \\
\hline 40 & 21.1469 & 0.3599 & 0.2782 & 0.1971 & 3.8310 & 1.481 & 6.267 & 0.176 & -2.45 & 0.24 & \\
\hline 42 & 21.0392 & 0.3932 & 0.3089 & 0.1697 & 3.6708 & 1.710 & 6.045 & 0.150 & -2.56 & 0.20 & $*$ \\
\hline 43 & 21.1188 & 0.3645 & 0.3324 & 0.2243 & 3.8744 & 1.791 & 6.075 & 0.151 & -1.94 & 0.20 & $*$ \\
\hline 45 & 21.1690 & 0.4129 & 0.3552 & 0.2593 & 3.8733 & 1.391 & 5.584 & 0.133 & -2.38 & 0.18 & $*$ \\
\hline 47 & 21.1743 & 0.3426 & 0.2996 & 0.1977 & 3.6999 & 1.895 & 6.150 & 0.178 & -1.96 & 0.24 & $*$ \\
\hline 49 & 21.1237 & 0.3706 & 0.2392 & 0.1690 & 3.6302 & 1.731 & 5.878 & 0.196 & -2.12 & 0.26 & $*$ \\
\hline 51 & 21.1322 & 0.3698 & 0.2734 & 0.1621 & 3.9393 & 1.626 & 5.700 & 0.161 & -2.19 & 0.22 & $*$ \\
\hline 52 & 21.1344 & 0.5317 & 0.3492 & 0.0578 & 4.3725 & 2.834 & 1.716 & 0.122 & -0.46 & 0.17 & \\
\hline 53 & 21.0354 & 0.5495 & 0.3102 & 0.1556 & 4.0508 & 2.151 & 0.046 & 0.237 & -1.65 & 0.32 & \\
\hline 54 & 21.1544 & 0.4462 & 0.2972 & 0.2325 & 3.7941 & 2.109 & 0.717 & 0.221 & -1.69 & 0.30 & \\
\hline 55 & 21.0562 & 0.3807 & 0.2762 & 0.2315 & 3.9886 & 1.590 & 5.568 & 0.178 & -2.21 & 0.24 & $*$ \\
\hline 56 & 21.1826 & 0.7219 & 0.7131 & 0.6050 & 4.0904 & 2.675 & 1.197 & 0.117 & -0.64 & 0.16 & \\
\hline 57 & 21.0546 & 0.4037 & 0.2996 & 0.1998 & 3.7945 & 1.673 & 6.110 & 0.203 & -2.12 & 0.28 & $*$ \\
\hline 58 & 21.1601 & 0.4425 & 0.3006 & 0.1511 & 3.8126 & 2.012 & 6.026 & 0.299 & -1.63 & 0.40 & $*$ \\
\hline 59 & 21.1954 & 0.3742 & 0.3120 & 0.1503 & 3.8208 & 1.539 & 5.581 & 0.111 & -2.22 & 0.15 & $*$ \\
\hline 60 & 21.0932 & 0.3856 & 0.3667 & 0.0409 & 4.5573 & 2.133 & 1.630 & 0.292 & -1.49 & 0.39 & \\
\hline 62 & 21.1604 & 0.2769 & 0.2547 & 0.1082 & 3.6509 & 1.119 & 0.357 & 0.249 & -2.89 & 0.34 & \\
\hline 63 & 21.1308 & 0.4094 & 0.3034 & 0.1444 & 3.8708 & 1.520 & 0.566 & 0.239 & -2.37 & 0.32 & \\
\hline 64 & 21.1639 & 0.4044 & 0.2060 & 0.1087 & 3.5979 & 1.046 & 4.927 & 0.336 & -2.97 & 0.46 & \\
\hline 65 & 21.1341 & 0.4056 & 0.3401 & 0.1892 & 3.7348 & 1.263 & 5.416 & 0.196 & -2.61 & 0.27 & $*$ \\
\hline 66 & 21.1917 & 0.4033 & 0.2293 & 0.1271 & 3.8670 & 1.352 & 1.749 & 0.227 & -2.81 & 0.31 & \\
\hline 69 & 21.1418 & 0.4391 & 0.3548 & 0.2247 & 3.8962 & 1.606 & 5.866 & 0.129 & -2.15 & 0.18 & $*$ \\
\hline 70 & 21.0688 & 0.4295 & 0.3121 & 0.2121 & 3.6864 & 1.545 & 5.568 & 0.161 & -2.41 & 0.22 & $*$ \\
\hline 74 & 21.1333 & 0.3436 & 0.3541 & 0.1874 & 3.8226 & 1.401 & 5.798 & 0.103 & -2.43 & 0.14 & \\
\hline 76 & 21.0668 & 0.5111 & 0.4381 & 0.3828 & 3.8159 & 1.663 & 5.946 & 0.135 & -2.01 & 0.18 & $*$ \\
\hline 80 & 21.1615 & 0.4577 & 0.3232 & 0.2484 & 4.0232 & 1.726 & 5.872 & 0.195 & -2.03 & 0.26 & $*$ \\
\hline 81 & 20.9603 & 0.4094 & 0.3117 & 0.1080 & 3.6554 & 2.259 & 2.538 & 0.239 & -2.01 & 0.32 & \\
\hline 82 & 21.1601 & 0.4776 & 0.2322 & 0.1698 & 3.8021 & 1.630 & 5.921 & 0.189 & -2.11 & 0.26 & $*$ \\
\hline 84 & 21.0138 & 0.3621 & 0.2656 & 0.2341 & 3.9118 & 1.392 & 5.785 & 0.140 & -2.44 & 0.19 & \\
\hline 85 & 21.1101 & 0.3688 & 0.3734 & 0.1821 & 3.8898 & 1.411 & 5.390 & 0.175 & -2.53 & 0.24 & $*$ \\
\hline 86 & 21.1539 & 0.4207 & 0.2335 & 0.0733 & 3.7654 & 1.913 & 6.089 & 0.337 & -1.91 & 0.45 & \\
\hline 87 & 21.2338 & 0.3278 & 0.4763 & 0.1339 & 4.5433 & 2.335 & 1.365 & 0.200 & -1.24 & 0.27 & \\
\hline 88 & 21.1992 & 0.4304 & 0.3728 & 0.2675 & 3.6537 & 1.639 & 5.915 & 0.181 & -2.15 & 0.25 & $*$ \\
\hline 89 & 21.1122 & 0.4373 & 0.2633 & 0.2134 & 3.9794 & 1.640 & 5.929 & 0.271 & -2.18 & 0.37 & $*$ \\
\hline 92 & 21.2012 & 0.4593 & 0.2218 & 0.2490 & 4.0976 & 1.786 & 5.667 & 0.245 & -1.73 & 0.33 & \\
\hline
\end{tabular}


Table 7

(Continued)

\begin{tabular}{|c|c|c|c|c|c|c|c|c|c|c|c|}
\hline ID & $A_{0}$ & $R_{21}$ & $R_{31}$ & $R_{41}$ & $\phi_{21}$ & $\phi_{31}$ & $\phi_{41}$ & $\sigma_{\phi_{31}}$ & {$[\mathrm{Fe} / \mathrm{H}]$} & $\sigma_{[\mathrm{Fe} / \mathrm{H}]}$ & $D_{M}$ Pass? \\
\hline 93 & 21.1188 & 0.3776 & 0.3039 & 0.2448 & 4.0668 & 1.558 & 5.937 & 0.132 & -2.17 & 0.18 & $*$ \\
\hline 94 & 21.1455 & 0.4514 & 0.2628 & 0.2586 & 3.7857 & 1.543 & 6.005 & 0.125 & -2.05 & 0.17 & \\
\hline 95 & 21.0371 & 0.3973 & 0.2391 & 0.1905 & 3.8537 & 1.621 & 5.608 & 0.176 & -2.26 & 0.24 & $*$ \\
\hline 98 & 21.0444 & 0.4845 & 0.3347 & 0.1981 & 3.8040 & 1.665 & 0.389 & 0.189 & -2.26 & 0.26 & \\
\hline 100 & 20.9654 & 0.3373 & 0.1896 & 0.0863 & 4.1915 & 1.908 & 1.242 & 0.299 & -2.57 & 0.40 & \\
\hline 101 & 21.1938 & 0.3419 & 0.4871 & 0.3298 & 3.9467 & 1.824 & 5.786 & 0.151 & -1.99 & 0.20 & $*$ \\
\hline 102 & 21.0912 & 0.3846 & 0.1889 & 0.2116 & 3.5606 & 1.948 & 0.430 & 0.187 & -1.60 & 0.25 & \\
\hline 103 & 21.1933 & 0.4730 & 0.3462 & 0.2441 & 3.8644 & 1.724 & 5.684 & 0.177 & -2.05 & 0.24 & $*$ \\
\hline 104 & 21.1413 & 0.3145 & 0.3785 & 0.2147 & 3.9168 & 1.419 & 5.685 & 0.111 & -2.40 & 0.16 & * \\
\hline 105 & 21.2349 & 0.3906 & 0.3318 & 0.1716 & 3.8405 & 1.935 & 6.225 & 0.147 & -1.79 & 0.20 & $*$ \\
\hline 107 & 21.1280 & 0.4623 & 0.3735 & 0.2258 & 3.5859 & 1.489 & 5.910 & 0.163 & -2.24 & 0.22 & $*$ \\
\hline 114 & 20.8940 & 0.3156 & 0.1729 & 0.1486 & 3.9526 & 1.759 & 6.230 & 0.237 & -2.23 & 0.32 & $*$ \\
\hline 115 & 21.0860 & 1.6991 & 1.7997 & 0.9826 & 2.0955 & 0.667 & 6.012 & 0.324 & -3.47 & 0.44 & \\
\hline 116 & 21.1802 & 2.2240 & 2.8269 & 0.6754 & 0.1569 & 1.101 & 4.885 & 3.001 & -3.35 & 4.04 & \\
\hline 119 & 21.1065 & 0.3107 & 0.2153 & 0.1698 & 4.1220 & 1.915 & 0.517 & 0.278 & -2.11 & 0.38 & \\
\hline 122 & 21.1773 & 0.3763 & 0.3510 & 0.1807 & 3.8666 & 1.947 & 0.367 & 0.369 & -1.92 & 0.50 & \\
\hline 124 & 21.1814 & 0.4343 & 0.2887 & 0.2845 & 3.7266 & 1.512 & 5.377 & 0.118 & -2.07 & 0.16 & \\
\hline 125 & 21.0576 & 0.3962 & 0.2374 & 0.1322 & 3.9208 & 1.807 & 0.055 & 0.143 & -2.36 & 0.19 & \\
\hline 126 & 21.1108 & 0.3502 & 0.3280 & 0.2460 & 3.6596 & 1.565 & 5.892 & 0.147 & -2.20 & 0.20 & $*$ \\
\hline 127 & 21.1686 & 0.3964 & 0.2482 & 0.1976 & 3.9177 & 1.577 & 6.208 & 0.142 & -2.59 & 0.20 & \\
\hline 128 & 21.1830 & 0.3977 & 0.3906 & 0.2593 & 3.6900 & 1.872 & 5.872 & 0.172 & -2.00 & 0.23 & * \\
\hline 129 & 21.2547 & 0.3290 & 0.1382 & 0.2019 & 4.1716 & 1.255 & 5.564 & 0.417 & -2.62 & 0.56 & \\
\hline 132 & 21.0987 & 0.1121 & 0.3401 & 0.1890 & 3.5035 & 2.589 & 6.112 & 0.166 & -0.98 & 0.23 & \\
\hline 133 & 21.0932 & 0.4467 & 0.2818 & 0.1231 & 3.8670 & 1.863 & 0.030 & 0.196 & -1.88 & 0.26 & \\
\hline 135 & 21.0948 & 0.4023 & 0.3453 & 0.1891 & 3.8332 & 1.757 & 5.891 & 0.117 & -2.15 & 0.16 & $*$ \\
\hline 136 & 21.1900 & 0.3180 & 0.2411 & 0.2928 & 3.9378 & 1.784 & 5.756 & 0.424 & -1.68 & 0.57 & \\
\hline 137 & 21.2138 & 0.3758 & 0.2625 & 0.1822 & 3.9266 & 1.589 & 5.710 & 0.168 & -2.22 & 0.23 & $*$ \\
\hline 140 & 21.0901 & 0.2879 & 0.1967 & 0.2204 & 2.3131 & 1.191 & 4.132 & 1.184 & -2.92 & 1.59 & \\
\hline 142 & 21.0906 & 0.4665 & 0.3385 & 0.2569 & 3.7860 & 1.634 & 5.772 & 0.102 & -2.36 & 0.14 & $*$ \\
\hline 144 & 21.3136 & 0.8180 & 0.2715 & 0.5360 & 2.4477 & 2.581 & 3.875 & 0.333 & -0.74 & 0.45 & \\
\hline 149 & 21.2231 & 0.3700 & 0.1774 & 0.1194 & 3.9287 & 3.235 & 0.367 & 0.394 & -0.31 & 0.54 & \\
\hline 150 & 21.0500 & 0.2377 & 0.1382 & 0.1382 & 4.3527 & 2.121 & 5.571 & 0.726 & -1.89 & 0.98 & \\
\hline 151 & 21.1498 & 0.3626 & 0.3154 & 0.0990 & 3.9220 & 1.622 & 0.008 & 0.209 & -2.28 & 0.28 & \\
\hline 152 & 21.1560 & 0.3985 & 0.3967 & 0.2306 & 3.6581 & 1.786 & 6.260 & 0.119 & -2.08 & 0.16 & $*$ \\
\hline 154 & 21.0598 & 0.3671 & 0.3369 & 0.1177 & 3.8109 & 1.313 & 5.592 & 0.158 & -2.78 & 0.22 & $*$ \\
\hline 159 & 21.0791 & 0.4384 & 0.2648 & 0.2246 & 4.0971 & 1.953 & 6.277 & 0.135 & -1.99 & 0.18 & $*$ \\
\hline 161 & 21.2265 & 0.3989 & 0.3224 & 0.2057 & 3.7740 & 1.729 & 6.173 & 0.143 & -2.13 & 0.19 & $*$ \\
\hline 162 & 21.1348 & 0.4299 & 0.2925 & 0.1395 & 3.9167 & 1.702 & 5.922 & 0.162 & -2.17 & 0.22 & $*$ \\
\hline 163 & 21.2684 & 0.4072 & 0.3391 & 0.2046 & 4.0556 & 2.067 & 6.148 & 0.314 & -1.31 & 0.42 & $*$ \\
\hline 164 & 21.1045 & 0.3637 & 0.3045 & 0.2021 & 3.6531 & 1.378 & 5.967 & 0.316 & -2.64 & 0.43 & $*$ \\
\hline 167 & 21.1274 & 0.3788 & 0.2584 & 0.2988 & 3.9965 & 1.796 & 0.072 & 0.203 & -2.30 & 0.28 & \\
\hline 171 & 21.1111 & 0.4379 & 0.2636 & 0.1877 & 3.8213 & 2.009 & 6.264 & 0.255 & -1.61 & 0.34 & $*$ \\
\hline 172 & 20.9743 & 0.3988 & 0.3147 & 0.1148 & 3.7654 & 1.898 & 0.471 & 0.124 & -2.13 & 0.17 & \\
\hline 174 & 20.9688 & 0.5443 & 0.2898 & 0.2588 & 4.2496 & 2.078 & 6.195 & 0.163 & -1.95 & 0.22 & $*$ \\
\hline 175 & 21.1595 & 0.4584 & 0.2483 & 0.1598 & 3.8483 & 1.593 & 5.813 & 0.151 & -1.99 & 0.20 & * \\
\hline 176 & 20.7044 & 0.4697 & 0.2381 & 0.1348 & 3.7171 & 1.321 & 5.613 & 0.214 & -2.60 & 0.29 & $*$ \\
\hline 177 & 21.0706 & 0.9609 & 0.5962 & 0.8721 & 5.1753 & 5.931 & 0.622 & 0.377 & 3.97 & 0.56 & \\
\hline 178 & 21.1075 & 0.3318 & 0.3033 & 0.2832 & 3.8723 & 1.613 & 0.042 & 0.133 & -2.14 & 0.18 & \\
\hline 180 & 21.0667 & 0.4906 & 0.3366 & 0.1060 & 3.9508 & 2.642 & 1.553 & 0.367 & -1.06 & 0.50 & \\
\hline 183 & 21.1632 & 0.4588 & 0.3604 & 0.2331 & 3.7826 & 1.490 & 5.939 & 0.153 & -2.32 & 0.21 & $*$ \\
\hline 185 & 21.2703 & 0.4016 & 0.2601 & 0.1861 & 3.8097 & 1.540 & 5.831 & 0.242 & -2.24 & 0.33 & $*$ \\
\hline 187 & 21.1778 & 0.6475 & 0.4987 & 0.3929 & 2.0980 & 2.169 & 3.850 & 0.154 & -1.89 & 0.21 & \\
\hline 196 & 21.2136 & 0.4445 & 0.3840 & 0.1823 & 3.8577 & 1.506 & 5.976 & 0.137 & -2.27 & 0.19 & $*$ \\
\hline 198 & 21.0808 & 0.3530 & 0.2225 & 0.0692 & 3.6715 & 1.804 & 1.183 & 0.255 & -2.35 & 0.34 & \\
\hline 199 & 21.1064 & 0.4746 & 0.3464 & 0.1720 & 4.0304 & 2.074 & 0.341 & 0.148 & -1.90 & 0.20 & \\
\hline 201 & 21.1134 & 0.3684 & 0.4381 & 0.2510 & 4.2339 & 1.746 & 0.049 & 0.216 & -2.32 & 0.29 & \\
\hline 207 & 21.1579 & 0.3866 & 0.3150 & 0.2058 & 3.6818 & 1.372 & 5.550 & 0.217 & -2.33 & 0.29 & $*$ \\
\hline 213 & 21.1666 & 0.4452 & 0.2605 & 0.1771 & 3.9542 & 2.009 & 5.510 & 0.276 & -1.75 & 0.37 & \\
\hline 216 & 21.1330 & 0.4461 & 0.3912 & 0.2201 & 3.3530 & 1.542 & 6.217 & 0.105 & -2.24 & 0.14 & \\
\hline 218 & 21.1418 & 0.4827 & 0.3112 & 0.2325 & 3.8122 & 1.236 & 4.587 & 0.253 & -2.75 & 0.34 & \\
\hline 219 & 21.2374 & 0.4351 & 0.3167 & 0.1716 & 3.9298 & 1.522 & 5.540 & 0.164 & -2.33 & 0.22 & $*$ \\
\hline 220 & 21.1473 & 0.4628 & 0.2067 & 0.1128 & 3.5244 & 1.388 & 5.629 & 0.286 & -2.62 & 0.39 & $*$ \\
\hline 223 & 21.2273 & 0.3467 & 0.3532 & 0.1648 & 3.9499 & 1.824 & 0.257 & 0.109 & -1.89 & 0.15 & \\
\hline 225 & 21.1668 & 0.3397 & 0.3088 & 0.1798 & 3.8777 & 1.429 & 5.352 & 0.268 & -2.30 & 0.36 & * \\
\hline 227 & 21.1436 & 0.4977 & 0.4236 & 0.2166 & 4.1281 & 1.515 & 0.083 & 0.167 & -2.32 & 0.23 & \\
\hline
\end{tabular}


Table 7

(Continued)

\begin{tabular}{|c|c|c|c|c|c|c|c|c|c|c|c|}
\hline ID & $A_{0}$ & $R_{21}$ & $R_{31}$ & $R_{41}$ & $\phi_{21}$ & $\phi_{31}$ & $\phi_{41}$ & $\sigma_{\phi_{31}}$ & {$[\mathrm{Fe} / \mathrm{H}]$} & $\sigma_{[\mathrm{Fe} / \mathrm{H}]}$ & $D_{M}$ Pass? $^{\mathrm{a}}$ \\
\hline 237 & 21.2335 & 0.4387 & 0.3462 & 0.1824 & 3.8619 & 1.578 & 5.537 & 0.127 & -2.30 & 0.17 & $*$ \\
\hline 238 & 21.0892 & 0.3513 & 0.2782 & 0.1435 & 3.6480 & 1.495 & 5.621 & 0.130 & -2.13 & 0.18 & $*$ \\
\hline 243 & 21.0025 & 0.2285 & 0.1968 & 0.1412 & 4.1500 & 2.210 & 1.336 & 0.314 & -2.02 & 0.42 & \\
\hline 244 & 21.2050 & 0.2709 & 0.1472 & 0.1502 & 3.9090 & 1.318 & 5.572 & 0.206 & -2.37 & 0.28 & $*$ \\
\hline 249 & 21.1367 & 0.3947 & 0.3224 & 0.2133 & 3.8850 & 1.689 & 5.687 & 0.086 & -2.07 & 0.12 & $*$ \\
\hline 252 & 21.1382 & 0.4386 & 0.3597 & 0.2268 & 3.8585 & 1.453 & 5.479 & 0.133 & -2.30 & 0.18 & $*$ \\
\hline 253 & 21.2784 & 0.2496 & 0.1984 & 0.5095 & 4.7083 & 4.776 & 4.581 & 0.357 & 2.34 & 0.51 & \\
\hline 258 & 21.0697 & 0.3559 & 0.3024 & 0.1948 & 3.7039 & 1.365 & 5.571 & 0.124 & -2.45 & 0.17 & $*$ \\
\hline 260 & 21.1105 & 0.5214 & 0.3487 & 0.2697 & 3.9380 & 1.576 & 5.989 & 0.085 & -1.98 & 0.12 & \\
\hline 261 & 21.1359 & 0.4000 & 0.2921 & 0.1263 & 3.7906 & 1.831 & 0.182 & 0.167 & -1.62 & 0.22 & \\
\hline 262 & 21.1091 & 0.3866 & 0.3278 & 0.1781 & 3.5986 & 1.408 & 6.122 & 0.092 & -2.56 & 0.13 & \\
\hline 265 & 21.0829 & 0.4074 & 0.2783 & 0.2220 & 3.7921 & 1.488 & 5.868 & 0.403 & -2.26 & 0.54 & $*$ \\
\hline 269 & 21.0926 & 0.4347 & 0.3432 & 0.2381 & 3.7428 & 1.413 & 5.332 & 0.127 & -2.14 & 0.17 & $*$ \\
\hline 270 & 20.9785 & 0.3460 & 0.3707 & 0.2696 & 3.7410 & 1.306 & 5.605 & 0.085 & -2.41 & 0.12 & $*$ \\
\hline 273 & 20.9756 & 0.0759 & 0.0814 & 0.1433 & 5.2331 & 2.817 & 1.126 & 0.859 & -1.55 & 1.16 & \\
\hline 278 & 21.1312 & 0.3491 & 0.3719 & 0.2490 & 3.7717 & 1.668 & 5.850 & 0.143 & -2.20 & 0.19 & $*$ \\
\hline 279 & 21.0067 & 0.4360 & 0.2030 & 0.1760 & 3.6151 & 1.206 & 5.978 & 0.223 & -2.79 & 0.31 & \\
\hline 281 & 20.8779 & 0.2710 & 0.6362 & 0.3831 & 1.8806 & 4.044 & 0.350 & 0.089 & 0.70 & 0.17 & \\
\hline 284 & 21.0144 & 0.1990 & 0.3959 & 0.2275 & 4.0643 & 1.113 & 5.740 & 0.228 & -2.92 & 0.31 & \\
\hline 285 & 20.8499 & 0.3775 & 0.2681 & 0.1193 & 4.1360 & 1.897 & 0.200 & 0.281 & -2.06 & 0.38 & \\
\hline 290 & 21.1048 & 0.4284 & 0.1804 & 0.1608 & 3.8519 & 1.824 & 1.108 & 0.491 & -2.48 & 0.66 & \\
\hline 291 & 20.7762 & 0.7227 & 0.5494 & 0.4493 & 3.8448 & 1.340 & 4.926 & 0.102 & -3.28 & 0.15 & \\
\hline 298 & 21.1788 & 0.3668 & 0.2674 & 0.2986 & 3.8061 & 0.882 & 4.773 & 0.257 & -3.42 & 0.35 & \\
\hline 303 & 21.2060 & 0.3931 & 0.3419 & 0.1996 & 4.3250 & 2.005 & 0.412 & 0.102 & -1.74 & 0.14 & \\
\hline 304 & 21.1033 & 0.4119 & 0.2785 & 0.1459 & 3.9121 & 1.802 & 5.678 & 0.234 & -2.27 & 0.32 & $*$ \\
\hline 308 & 21.0490 & 0.3422 & 0.0718 & 0.2947 & 3.0191 & 0.890 & 4.789 & 0.953 & -3.24 & 1.28 & \\
\hline 309 & 21.0466 & 0.3957 & 0.2182 & 0.2359 & 3.2974 & 1.333 & 5.126 & 0.208 & -2.68 & 0.28 & \\
\hline 310 & 21.0316 & 0.4385 & 0.3614 & 0.3383 & 4.1751 & 2.114 & 0.496 & 0.170 & -1.74 & 0.23 & \\
\hline 313 & 21.0945 & 0.3778 & 0.2171 & 0.1559 & 3.8761 & 1.283 & 4.963 & 0.184 & -2.48 & 0.25 & $*$ \\
\hline 315 & 21.0593 & 0.4135 & 0.1561 & 0.1555 & 3.5577 & 1.364 & 0.402 & 0.316 & -2.41 & 0.43 & \\
\hline 323 & 21.0327 & 0.3434 & 0.2483 & 0.2011 & 3.9195 & 1.239 & 5.380 & 0.153 & -2.45 & 0.21 & $*$ \\
\hline 324 & 21.0505 & 0.4123 & 0.2956 & 0.1909 & 3.7772 & 1.367 & 5.935 & 0.132 & -2.52 & 0.18 & \\
\hline 325 & 21.0045 & 0.2638 & 0.3611 & 0.1385 & 4.5856 & 2.350 & 0.748 & 0.269 & -1.27 & 0.36 & \\
\hline 326 & 21.0456 & 0.4710 & 0.2458 & 0.2248 & 3.4030 & 1.104 & 5.710 & 0.160 & -3.02 & 0.22 & \\
\hline 332 & 21.1832 & 0.2445 & 0.3541 & 0.4182 & 3.7120 & 0.430 & 5.168 & 0.443 & -3.91 & 0.60 & \\
\hline
\end{tabular}

Note. ${ }^{\text {a }}$ Stars with an asterisk $(*)$ have passed the $D_{M}<5.0$ criterion.

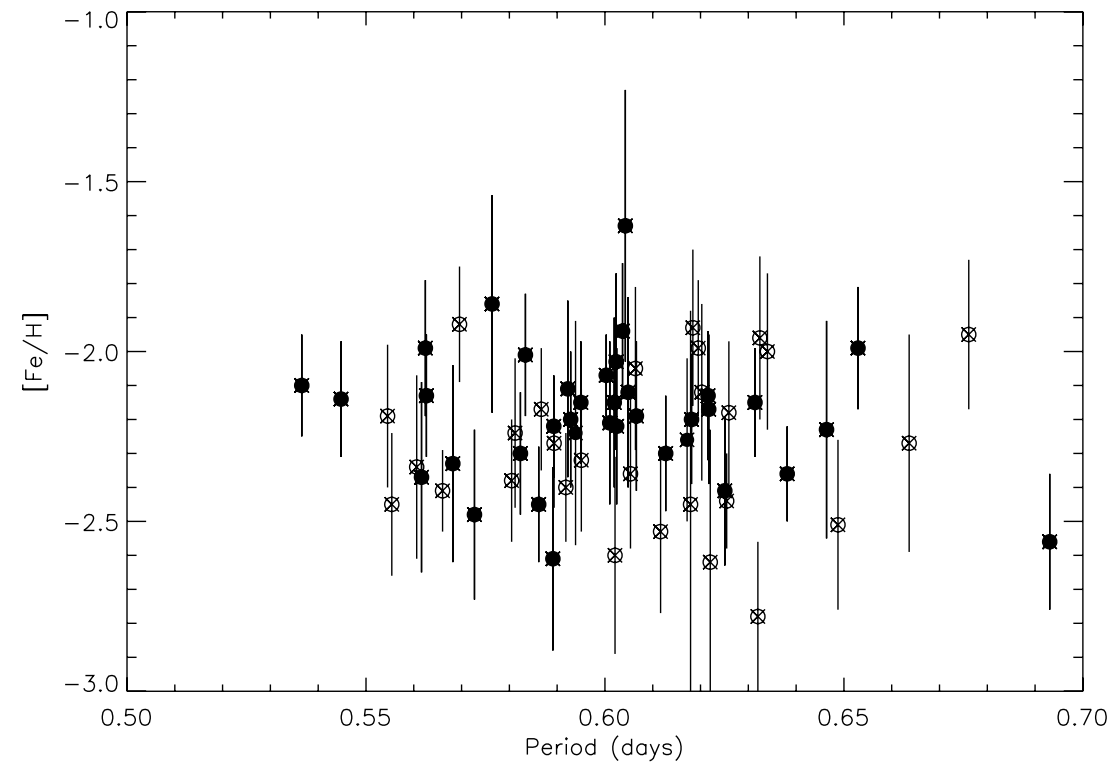

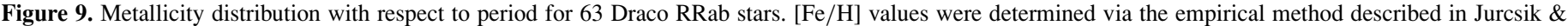
Kovacs (1996). The filled points correspond to $D_{M}<3.0$ and the open points are for $3.0<D_{M}<5.0$. 
Table 8

Parameters for Draco ACs

\begin{tabular}{cccccccc}
\hline \hline ID $^{\mathrm{a}}$ & $\begin{array}{c}\text { R.A. } \\
(2000.0)\end{array}$ & $\begin{array}{c}\text { Decl. } \\
(2000.0)\end{array}$ & $\begin{array}{c}\text { Period } \\
\text { (days) }\end{array}$ & Amplitude & $\langle V\rangle$ & $\langle I\rangle$ & $M_{V}$ \\
\hline 31 & $17: 20: 25.15$ & $57: 52: 53.3$ & 0.61763 & 0.71 & 19.57 & 18.78 & -0.01 \\
$134^{*}$ & $17: 19: 06.37$ & $57: 49: 48.2$ & 0.59228 & 0.85 & 18.78 & 18.40 & -0.80 \\
$141^{*}$ & $17: 20: 17.82$ & $57: 57: 07.8$ & 0.90087 & 0.67 & 19.20 & 18.63 & -0.38 \\
$157^{*}$ & $17: 19: 08.08$ & $57: 58: 35.2$ & 0.93649 & 1.04 & 18.85 & 18.41 & -0.74 \\
$194 *$ & $17: 19: 36.06$ & $57: 54: 15.6$ & 1.59027 & 0.48 & 18.11 & 17.53 & -1.47 \\
$204^{*}$ & $17: 22: 00.74$ & $57: 50: 21.2$ & 0.45413 & 0.75 & 19.23 & 18.77 & -0.35 \\
230 & $17: 21: 47.77$ & $57: 53: 18.9$ & 0.60816 & 0.38 & 19.25 & 18.54 & -0.33 \\
282 & $17: 19: 42.55$ & $57: 54: 49.8$ & 0.55187 & 0.60 & 19.51 & 18.90 & -0.05 \\
312 & $17: 18: 30.56$ & $57: 56: 04.8$ & 0.90735 & 0.90 & 19.15 & 18.59 & -0.43 \\
\hline
\end{tabular}

Note. ${ }^{\text {a }}$ Stars denoted with an asterisk (*) are previously known ACs (Norris \& Zinn 1975; Zinn \& Searle 1976; Nemec et al. 1988).

our data. Furthermore, most are either sufficiently bright or have large amplitudes that they cannot be an RRL star made brighter by an unresolved companion. However for V31, V230, and V282, we cannot exclude this possibility of RRL-plusunresolved companion. In Figure 10, we present the light curves of all the ACs found in this survey with a spline fit added to aid the eye.

Generally, these variable stars are brighter than the RRL population by 0.5 (for shorter period, $P \sim 0.3$ days) to $2 \mathrm{mag}$ (for longer period, $P \sim 2.0$ days). These stars are also more massive than the RRL, typically 1.0-2.0 $M_{\odot}$ (Pritzl et al. 2002a, and references therein), and must be relatively metal poor in order for the progenitor stars to reach the instability strip. ACs have been found in all the known $\mathrm{dSph}$ galaxies of the Local Group, however, they are not typically found in the Galactic globular clusters. The exceptions are V19 in NGC 5466 (Zinn $\&$ Dahn 1976) and two candidates in $\omega$ Cen (Wallerstein \& Cox 1984). XZ Ceti is a well-known field AC. The origins of these stars still remains unsolved, but the leading theories suggest that they are either (1) intermediate-aged stars $(t<5 \mathrm{Gyr})$ or (2) primordial binary systems undergoing mass transfer. These mechanisms provide alternative origins for the blue straggler populations that have been speculated to be the progenitor stars of the AC.

Recently, Momany et al. (2007) investigated the frequency of blue straggler stars in the Local Group dSph population, compared with the frequency of such stars in Galactic globular clusters, open clusters, and the field. They find that, in general, the blue straggler frequency is higher in $\mathrm{dSph}$ galaxies than in globular clusters. If the blue straggler stars are progenitors of the ACs, then this higher frequency is consistent with a higher frequency of ACs among the dSph systems. It is noteworthy, too, that some mechanisms for creating blue stragglers by mass transfer may not operate in systems of low stellar density, such as the dSph. For example, it has been suggested that collisional binary systems might create blue straggler stars, but such collisions would be infrequent in dSph systems (Momany et al. 2007). Thus, to consider the blue straggler star frequency, one must only consider those stellar formation mechanisms that will be likely in a dSph environment if one wishes to correlate the frequency with the number of AC stars found.

ACs of dSph galaxies have also been used to create a period-luminosity (P-L) relation. Recent work by Dall'Ora et al. (2003), Pritzl et al. (2002a), and Nemec et al. (1994) have presented empirical AC $\mathrm{P}-\mathrm{L}$ relations associated with the pulsational mode. Both empirical and theoretical $\mathrm{P}-\mathrm{L}$ relations have shown that they are not parallel (Pritzl et al. 2002a; Bono et al. 1997). However, there is still some question as to whether the two apparent $\mathrm{P}-\mathrm{L}$ relations are real, due to distinct fundamental and first-overtone mode relations, or whether the results might instead be interpreted as a single $\mathrm{P}-\mathrm{L}$ relation with large scatter. That scatter might be a reflection of the range of $\mathrm{AC}$ masses as well as the finite width of the instability strip.

For the Draco AC sample, we applied the empirical $\mathrm{P}-\mathrm{L}$ relations of Pritzl et al. (2002a) to see whether the location of the additional Draco stars would support the reality of two distinct $\mathrm{P}-\mathrm{L}$ relations. We have calculated absolute magnitudes for the Draco AC stars assuming a distance modulus of $(m-M)_{0}=$ 19.49 and an $E(B-V)=0.03$ (Pritzl et al. 2002a) in order to incorporate our results with their empirical $\mathrm{P}-\mathrm{L}$ relations. Figure 11 shows the location of the Draco AC stars with respect to the AC stars found in other Local Group dwarf galaxies. We see that most of the Draco ACs (V31, V141, V157, V194, V230, V282, and V312) fall along the P-L relation for stars pulsating in the fundamental mode, but two, V134 and V204, fall closer to the first-overtone mode $\mathrm{P}-\mathrm{L}$ relation. As discussed in Pritzl et al. (2002a), it is difficult to assign the pulsational mode in this manner, especially if phase coverage is not complete. We find this to be the case for the Draco ACs as well. Two possible firstovertone pulsators, V134 and V204, have light curves showing only modest asymmetry. Among RRL stars, that is a sign of RRc or first-overtone mode pulsation. However, the light curves for the supposed fundamental mode pulsator, V194, seem similar. Thus, we can only indicate that while there is evidence in Figure 11 for two distinct AC $\mathrm{P}-\mathrm{L}$ relations, the actual situation is still uncertain. For example, a range of masses among the ACs might influence the positions of the Draco AC within the $\mathrm{P}-\mathrm{L}$ diagram, and it perhaps cannot be entirely excluded that a single $\mathrm{P}-\mathrm{L}$ relation with scatter could account for the observations.

\subsection{Other Variable Stars}

Four categories of variable stars other than RRLs and Cepheids appear in our data: two eclipsing binaries, 30 "bluish long-period variables," 12 red semi-regular or irregular variables, and carbon stars have been found and are listed in Tables 9 and 10. The following subsections discuss each of these types of stars.

\subsubsection{Eclipsing Binary Stars}

A field eclipsing binary star (V296) was found in the survey completed by Bonanos et al. (2004), which we have recovered in our work. We agree with their period solution for this star, with a period of 0.2435 days. Figure 12 shows the light curve of V296 phased to this period. Additionally, we have also found another possible eclipsing binary star with a small amplitude change. This star, V256, has few faint observations, and our period result is somewhat uncertain. In Table 9, we provide two plausible period solutions. However, to truly confirm the nature of this eclipsing binary, a careful follow-up will be needed to arrive at the correct period.

\subsubsection{Carbon Stars}

A population of stars redward of the RGB has been often identified as carbon stars (Aaronson et al. 1983). There are six carbon stars known in Draco (C1-C3: Aaronson et al. 1982); (C4: Azzopardi et al. 1986); (C5: Armandroff et al. 1995); (C6: Shetrone et al. 2001b). We find the stars C1, C2, and C5 to be variable with $V$ amplitudes close to $0.2 \mathrm{mag}$. Stars C3, C4, and 

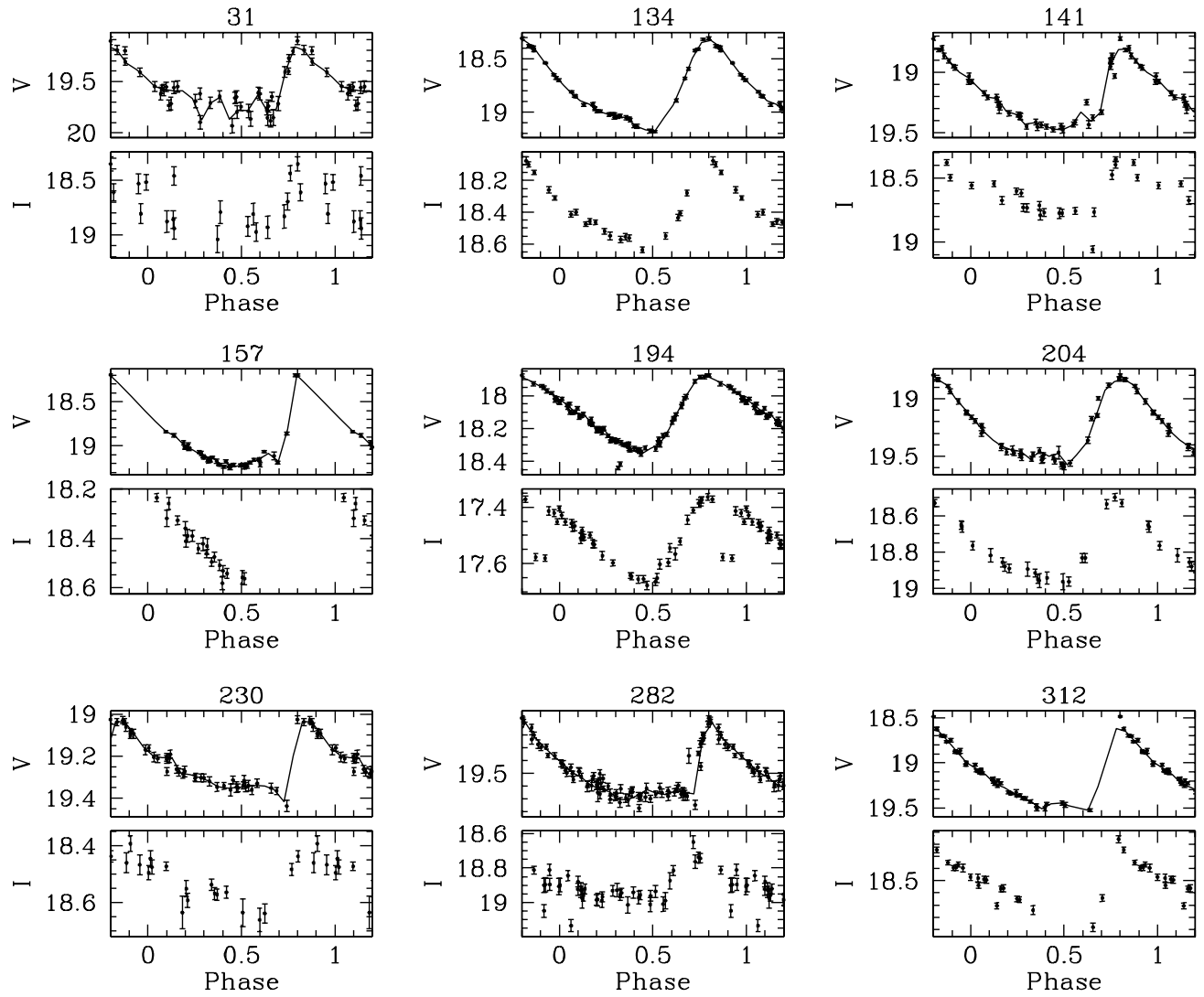

Figure 10. Phased light curves of the Draco ACs.

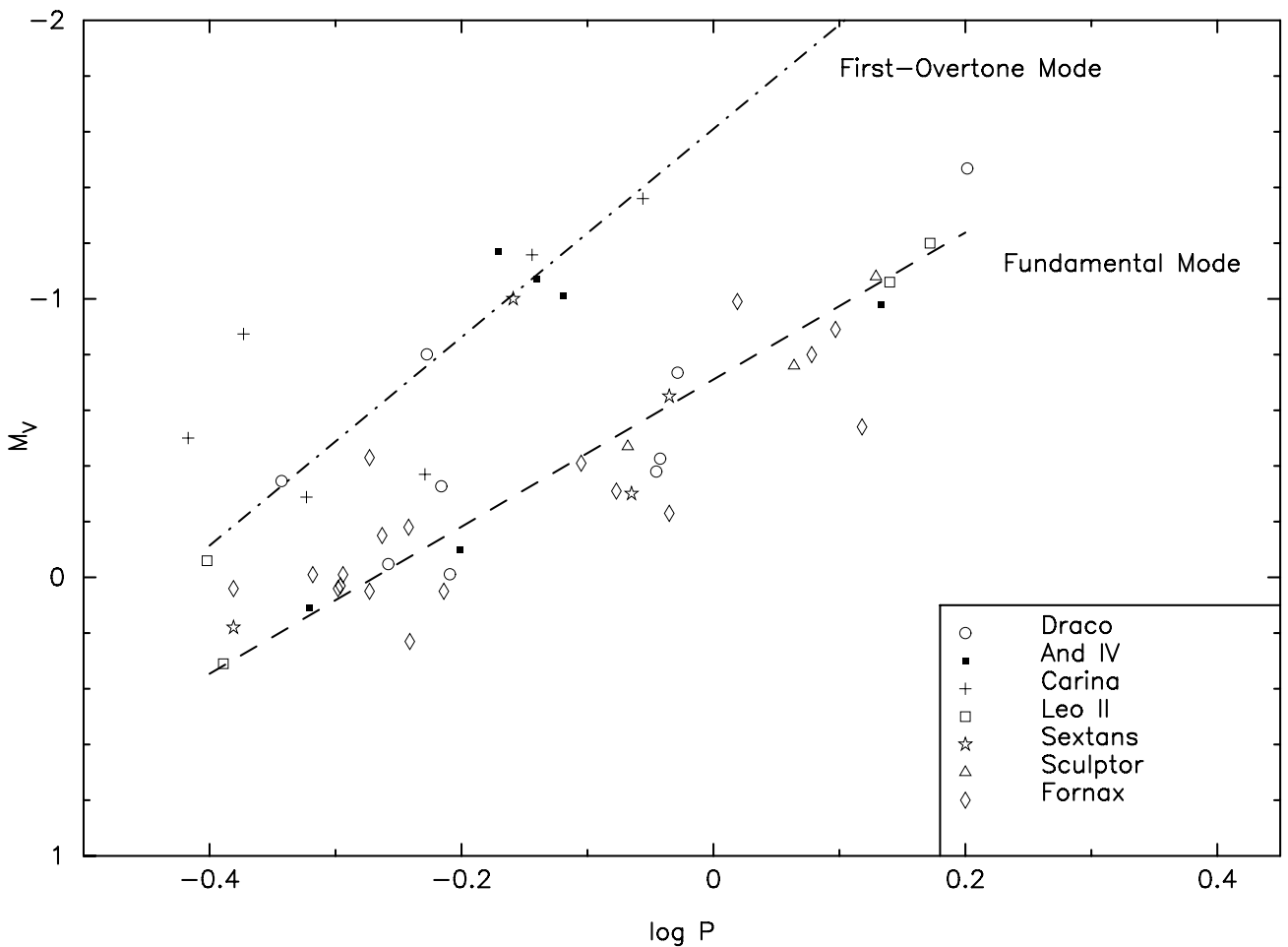

Figure 11. Draco ACs with respect to other ACs found in dSph galaxies. Information of ACs of other dwarf galaxies are from Pritzl et al. (2002a) and Dall'Ora et al. (2003). P-L relations for the fundamental and first-overtone pulsational modes from Pritzl et al. (2002a) are included.

C6 do not appear to vary during two seasons of observations at USNO. Shetrone et al. (2001b) also reported C2 as a definite variable and $\mathrm{C} 5$ as a possible variable.
The unusual nature of star $\mathrm{C} 1$ was noted by Aaronson et al. (1982) and by Margon et al. (2002) from their independent study of the star in a spectrum from the Sloan Digital Sky Survey 


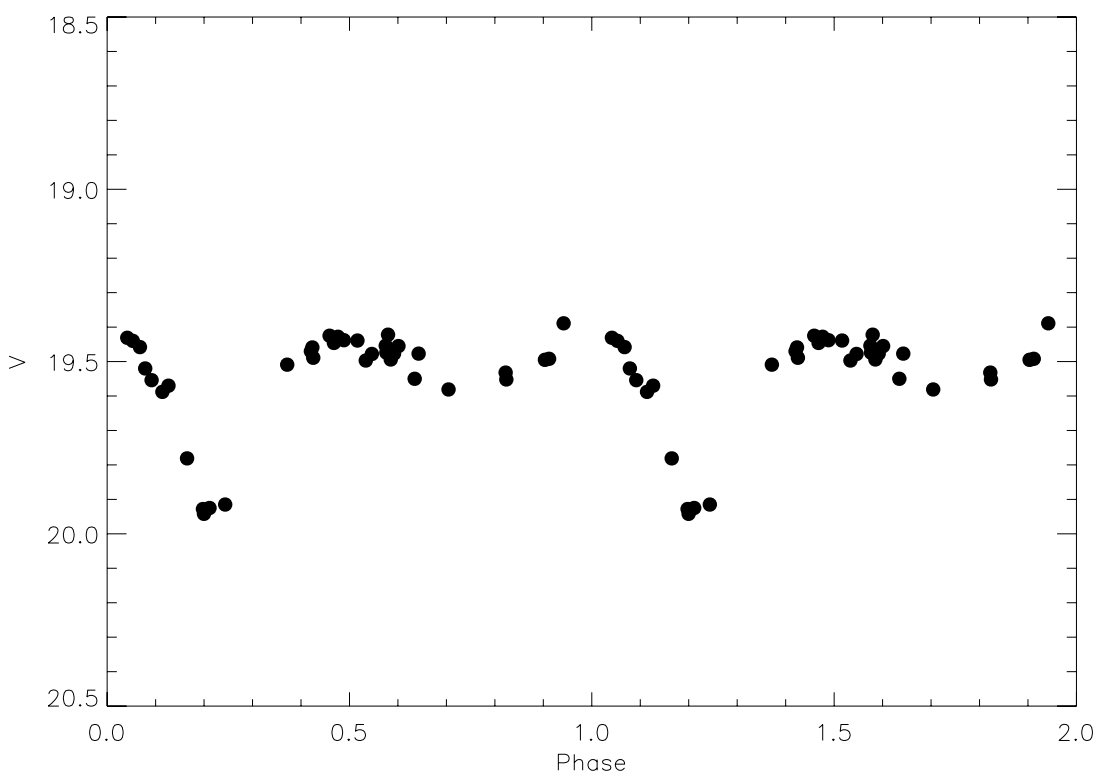

Figure 12. Field eclipsing binary star found in Bonanos et al. (2004) (their ID: J171906.2+574120.9) and in this work (our ID: V296). Period is 0.2435 days, which agrees well with their derived period.

Table 9

Long-Period, Semi-regular Red Variable Stars, and Carbon Stars

\begin{tabular}{cccccccccc}
\hline \hline ID & $\begin{array}{c}\text { R.A. } \\
(2000.0)\end{array}$ & $\begin{array}{c}\text { Decl. } \\
(2000.0)\end{array}$ & $V$ & $I$ & $V-I$ & $\sigma_{V}$ & $\sigma_{I}$ & $\begin{array}{c}\text { Reference/ } \\
\text { Comment }\end{array}$ \\
\hline 302 & $17: 18: 52.12$ & $58: 04: 13.2$ & 17.26 & 15.88 & 1.38 & 0.04 & 0.03 & 1 \\
293 & $17: 19: 10.82$ & $57: 59: 17.7$ & 17.14 & 15.76 & 1.39 & 0.03 & 0.01 & 1 \\
292 & $17: 19: 17.52$ & $58: 01: 07.4$ & 16.84 & 15.35 & 1.48 & 0.06 & 0.03 & 1,2 var. vel. \\
288 & $17: 19: 42.39$ & $57: 58: 38.0$ & 17.25 & 15.99 & 1.26 & 0.07 & 0.08 & 1 carbon star $($ C5) \\
283 & $17: 19: 57.66$ & $57: 50: 05.7$ & 17.15 & 15.66 & 1.49 & 0.08 & 0.04 & 1,2 carbon star $($ C 1$)$ var. vel. \\
280 & $17: 20: 00.70$ & $57: 53: 46.8$ & 17.30 & 15.99 & 1.31 & 0.05 & 0.04 & $1,2,3$ carbon star $(\mathrm{C} 2)$ \\
274 & $17: 20: 32.85$ & $57: 51: 44.2$ & 16.91 & 15.38 & 1.53 & 0.06 & 0.05 & $1,2,3$ \\
272 & $17: 20: 40.26$ & $57: 57: 33.1$ & 16.44 & 14.90 & 1.55 & 0.02 & 0.02 & 1,3 \\
271 & $17: 20: 41.85$ & $58: 00: 25.1$ & 16.93 & 15.44 & 1.48 & 0.05 & 0.02 & 1,2 \\
268 & $17: 20: 43.69$ & $57: 48: 44.3$ & 16.51 & 15.03 & 1.48 & 0.04 & 0.03 & 1,2 var. vel \\
263 & $17: 20: 53.01$ & $57: 55: 58.0$ & 17.23 & 15.70 & 1.53 & 0.02 & 0.02 & $1,2,3$ \\
259 & $17: 21: 02.23$ & $58: 15: 38.7$ & 17.15 & 15.75 & 1.41 & 0.04 & 0.03 & 1 \\
& Eclipsing Binaries & & & & & & & \\
296 & $17: 19: 06.16$ & $57: 41: 21.1$ & 19.54 & 18.16 & 1.38 & 0.15 & 0.12 & $P=0.2435$ days \\
256 & $17: 21: 18.30$ & $58: 14: 29.9$ & 18.54 & 17.28 & 1.26 & 0.03 & 0.10 & $P=0.1253$ days or 0.2300 days
\end{tabular}

References. 1. Draco RV member (Armandroff et al. 1995); 2. Draco RV member (Olszewski et al. 1995); 3. Draco proper motion member (Stetson 1980).

(SDSS). The strong emission lines of hydrogen and helium, the blue continuum flux, and the X-ray emission indicate that it is a symbiotic carbon star like UV Aur. Therefore, its photometric variability is not surprising. It also has a variable radial velocity (Olszewski et al. 1995) that is likely to be caused by orbital motion and may be independent of its variable brightness. The other carbon stars, including the two that we find to be variable, have not shown variable velocities.

\subsubsection{Long-Period Variables and QSOs}

The characteristics of the bluish long-period variables are slow variability, no apparent period, amplitudes typically of $0.25 \mathrm{mag}$, colors blueward of the Draco giant branch, and no clear concentration toward Draco. These characteristics suggest that most of them are background QSOs, and this hypothesis has been supported by available spectroscopy. The red semiregular variables have colors and magnitudes placing them near the tip of the RGB in Draco, and they all have radial velocities and/or proper motions showing they are members of Draco. Figure 13 shows our time series photometric data of the red long-period variable stars. Spline fits were not included since we assume that the coverage of the full variation was not obtained through the time coverage of our data set. Also, due to the approximately $40 \mathrm{~V}$ observations, we cannot provide robust estimations in the amplitudes of these long-period variables. In Table 9, we list mean magnitudes rather than intensity-weighted mean magnitudes due to our spotty phase coverage and the low amplitudes of these objects.

$\mathrm{B} \& \mathrm{~S}$ remarked on the lack of red variables found in Draco. Bonanos et al. (2004) showed that there are variables among the stars near the tip of the giant branch, as also shown in Figure 2. Our 12 red variables are mostly of low amplitude, and the amplitudes must have been just below the threshold for detection by B\&S. We now know that in metal-poor systems like Draco, 
Table 10

QSOs Found in Our Draco Survey

\begin{tabular}{|c|c|c|c|c|c|c|c|c|}
\hline ID & $\begin{array}{c}\text { R.A. } \\
(2000.0)\end{array}$ & $\begin{array}{c}\text { Decl. } \\
(2000.0)\end{array}$ & $V$ & $I$ & $V-I$ & $\sigma_{V}$ & $\sigma_{I}$ & Comment \\
\hline 331 & $17: 17: 35.09$ & $57: 56: 26.2$ & 19.66 & 18.98 & 0.67 & 0.07 & 0.06 & WIYN \\
\hline 329 & $17: 17: 50.11$ & $58: 11: 08.1$ & 19.96 & 19.13 & 0.83 & 0.08 & 0.06 & Probable QSO \\
\hline 328 & $17: 17: 50.57$ & $58: 15: 14.7$ & 17.71 & 16.92 & 0.79 & 0.06 & 0.06 & SDSS \\
\hline 322 & 17:18:09.35 & $58: 07: 16.3$ & 19.86 & 19.44 & 0.42 & 0.07 & 0.07 & WIYN \\
\hline 320 & $17: 18: 19.40$ & $57: 39: 35.2$ & 20.18 & 19.30 & 0.87 & 0.15 & 0.06 & WIYN \\
\hline 311 & $17: 18: 31.90$ & $58: 08: 44.3$ & 19.17 & 18.85 & 0.32 & 0.14 & 0.04 & WIYN \\
\hline 300 & 17:19:01.71 & 58:00:29.1 & 19.31 & 18.60 & 0.71 & 0.06 & 0.02 & WIYN, SDSS \\
\hline 299 & 17:19:04.18 & $58: 03: 29.4$ & 20.36 & 19.88 & 0.48 & 0.11 & 0.07 & WIYN \\
\hline 203 & $17: 19: 34.43$ & $57: 58: 49.8$ & 19.51 & 18.80 & 0.71 & 0.20 & 0.10 & WIYN $^{\mathrm{a}}$ \\
\hline 287 & 17:19:43.77 & $58: 11: 12.4$ & 19.87 & 18.85 & 1.01 & 0.06 & 0.04 & SDSS \\
\hline 266 & $17: 20: 51.96$ & $57: 41: 59.9$ & 19.52 & 18.99 & 0.53 & 0.07 & 0.04 & Probable QSO \\
\hline 264 & $17: 20: 52.31$ & $57: 55: 13.4$ & 19.86 & 19.43 & 0.40 & 0.08 & 0.06 & WIYN \\
\hline 257 & $17: 21: 18.12$ & $57: 33: 30.5$ & 20.25 & 19.19 & 1.05 & 0.17 & 0.08 & Probable QSO \\
\hline 255 & $17: 21: 22.85$ & $57: 50: 29.5$ & 17.90 & 17.26 & 0.64 & 0.02 & 0.01 & WIYN, SDSS \\
\hline 254 & $17: 21: 25.49$ & $58: 15: 28.2$ & 20.34 & 19.65 & 0.69 & 0.08 & 0.05 & SDSS \\
\hline 251 & $17: 21: 30.06$ & $57: 40: 15.8$ & 19.69 & 19.15 & 0.54 & 0.06 & 0.05 & WIYN \\
\hline 240 & $17: 21: 41.47$ & $57: 52: 35.6$ & 20.65 & 19.83 & 0.82 & 0.09 & 0.08 & Probable QSO \\
\hline 241 & $17: 21: 41.57$ & $57: 33: 18.9$ & 18.88 & 18.24 & 0.64 & 0.06 & 0.17 & WIYN, SDSS \\
\hline 231 & $17: 21: 47.52$ & $58: 15: 07.8$ & 19.96 & 18.46 & 1.50 & 0.14 & 0.04 & WIYN \\
\hline 229 & $17: 21: 48.30$ & $57: 58: 05.8$ & 20.70 & 19.75 & 0.95 & 0.11 & 0.07 & SDSS \\
\hline 224 & 17:22:07.34 & $58: 14: 25.0$ & 20.79 & 20.25 & 0.54 & 0.25 & 0.14 & PROBABLE QSO \\
\hline 222 & $17: 22: 11.66$ & $57: 56: 52.2$ & 20.74 & 19.77 & 0.98 & 0.16 & 0.09 & WIYN, SDSS \\
\hline 215 & $17: 22: 36.06$ & $57: 37: 05.0$ & 20.10 & 19.90 & 0.21 & 0.19 & 0.08 & WIYN \\
\hline 214 & $17: 22: 41.04$ & $57: 45: 27.1$ & 20.78 & 19.94 & 0.83 & 0.10 & 0.07 & PROBABLE QSO \\
\hline 212 & $17: 22: 44.68$ & $57: 41: 24.2$ & 20.40 & 19.47 & 0.93 & 0.15 & 0.08 & Probable QSO \\
\hline 211 & 17:22:51.01 & $57: 41: 18.5$ & 19.80 & 19.27 & 0.53 & 0.14 & 0.06 & WIYN \\
\hline 210 & $17: 22: 56.95$ & $58: 11: 10.8$ & 19.76 & 19.01 & 0.75 & 0.14 & 0.08 & WIYN, SDSS \\
\hline 209 & $17: 23: 01.83$ & $58: 04: 06.7$ & 20.52 & 19.10 & 1.42 & 0.14 & 0.07 & Probably QSO ${ }^{b}$ \\
\hline 208 & $17: 23: 02.20$ & 58:04:14.5 & 20.17 & 19.45 & 0.71 & 0.08 & 0.06 & WIYN \\
\hline 206 & 17:23:14.18 & 58:14:07.4 & 19.99 & 19.57 & 0.42 & 0.17 & 0.09 & WIYN \\
\hline
\end{tabular}

Notes. The last column lists where the spectra, if available, were obtained.

a B\&S V203.

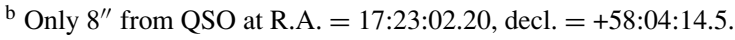

high-amplitude red variables like Miras are absent, and lowamplitude semi-regular or irregular variables are more common (e.g., Harris 1987).

Distinguishing between background QSOs and red variables in Draco is not always obvious, however, because QSOs sometimes can be red, and some Draco variables might be bluish and without regular periods. For example, UU Her and RV Tauri stars are found in globular clusters and could be confused here with our limited data. Therefore, spectroscopy is useful to ensure accurate classification: 22 bluish long-period variables are confirmed as QSOs with spectra taken with the WIYN telescope and described in a separate paper (H. C. Harris \& J. A. Munn 2008, in preparation), and/or spectra from the SDSS that put them in the SDSS DR3 QSO Catalog (Schneider et al. 2005). Eight additional variables with similar characteristics are listed in Table 10 as probable QSOs.

The prototype of the QSOs behind Draco, V203, was found by $B \& S$ and given in their Table VII, and the light curve spanning over six years was shown in their Figure 8. They did not understand its nature, but their light curve was the best study of variability of a QSO at that time. Of course, the redshift of QSOs and their characteristic variability were not discovered for two more years (Schmidt 1963; Matthews \& Sandage 1963).

\section{DRACO AND THE OOSTERHOFF DICHOTOMY}

Oosterhoff (1939) found that five RRL-rich globular clusters could be divided into two groups, now known as Oosterhoff groups, on the basis of the properties of their RRL stars. Subsequent investigations found that almost all of the Milky Way globular clusters that contain significant numbers of RRL stars could be placed into one or the other of the Oosterhoff groups. The RRL in Oosterhoff group I clusters have $\left\langle P_{a b}\right\rangle \sim$ 0.55 and $\left\langle P_{c}\right\rangle \sim 0.32$. RRL in Oosterhoff II clusters have $\left\langle P_{a b}\right\rangle \sim 0.64$ and $\left\langle P_{c}\right\rangle \sim 0.37$. Oosterhoff II clusters are also relatively richer in RRc variables than Oosterhoff I clusters, and they are more metal poor than Oosterhoff I clusters (see, for example, Smith 1995). However, not all systems show the Oosterhoff dichotomy. In contrast to the Milky Way globular clusters, dSph systems often have Oosterhoff intermediate properties (for recent discussions, see Pritzl et al. 2002a; Catelan 2004; Catelan 2005).

The mean period of RRab stars in Draco found here, 0.615 , seems to confirm its Oosterhoff intermediate nature. However, a detailed inspection of the properties of its RRL suggests a complicated picture. The Draco period-amplitude (Bailey) diagram (Figure 14) is consistent with an Oosterhoff intermediate classification. Many of the RRab stars occupy positions in this diagram close to that of the Clement et al. (2001) Oosterhoff I line, but many also fall to the right of that line, in the Oosterhoff intermediate zone. This result is qualitatively true if the trend lines of Cacciari et al. (2005) are used instead of those of Clement \& Rowe (2000). The Cacciari et al. (2005) lines are based on the period-amplitude distribution of RRab, some of which are evolved along the HB, of M3. In the Milky Way, a 

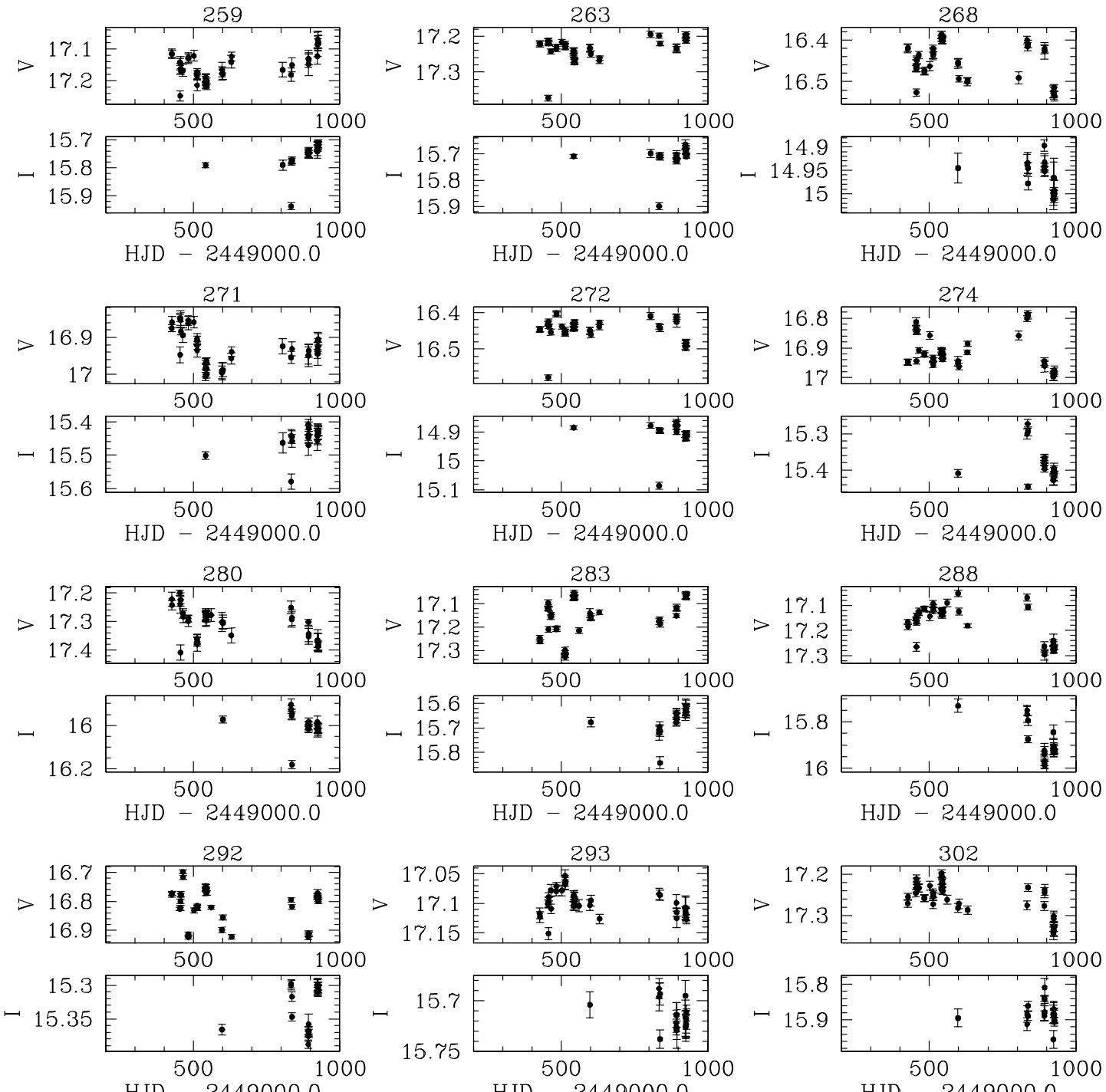

Figure 13. Time series data of the long-period variable stars found in our survey. The $x$-axis is the Heliocentric Julian Date (HJD-2449000.0).

metallicity of $[\mathrm{Fe} / \mathrm{H}]=-2.1$ would be typical of globular clusters of Oosterhoff type II. However, the ratio of RRcd to RRab stars in Draco, 0.26, is typical of Oosterhoff type I clusters. In contrast, the RRcd stars in Draco appear dominated by stars having Oosterhoff type II characteristics. This is particularly true of the RRd stars - all but one of which fall in the Petersen diagram in the region occupied by double-mode stars observed in Oosterhoff II clusters, such as M15. In summary, RRab stars in the period-amplitude diagram and the mean RRab period suggest an Oosterhoff intermediate class. However, the RRcd population has attributes usually associated with an Oosterhoff type II system. The mean period of the RRcd stars, 0.39 , is long and the location of the RRd stars in the Petersen diagram suggests a mainly Oosterhoff II class. Figure 5 shows a sharp fall off in the number of RRcd stars as one goes to shorter periods. This in part reflects an overall falloff in the number of $\mathrm{HB}$ stars as one goes from red to blue across the instability strip. There is a hint of a bimodal distribution in the RRc periods, but its significance is uncertain because of the small number of RRc stars toward the short-period end of the distribution. Conclusions as to the Oosterhoff classification of the RRL stars are probably more certain when based upon the more numerous RRab variable stars.
It is plausible that the discordant Oosterhoff properties of the Draco RRL are in some way related to the overall distribution of stars across its HB. Draco has a HB that is redder than those found among ordinary Oosterhoff II clusters, or among Milky Way globular clusters having $[\mathrm{Fe} / \mathrm{H}]=-2$ (see, for example, Catelan 2005). It has been proposed (Lee et al. 1990; Clement \& Shelton 1999; Clement et al. 2001) that many RRL in Oosterhoff type II systems have evolved into the blue part of the instability strip from zero-age horizontal-branch (ZAHB) positions. The paucity of blue HB stars in Draco makes it unlikely that the majority of its RRL have evolved from ZAHB positions on the blue HB, and thus, perhaps it is not surprising that Draco is not an Oosterhoff type II system despite having a low value of $[\mathrm{Fe} / \mathrm{H}]$. There may, however, be problems with the hypothesis that Oosterhoff II RRL are evolved blue HB stars. Even in the cases of ordinary Oosterhoff type II globular clusters, it has been argued that, according to conventional stellar evolutionary theory, the HB stars evolving from the blue HB would not spend sufficient time in the instability strip to produce the observed numbers of RRL (Renzini \& Fusi Pecci 1988; Rood \& Crocker 1989; Pritzl et al. 2002b). Thus, the exact role played by Draco's redder $\mathrm{HB}$ in producing its confusing Oosterhoff properties remains uncertain. 


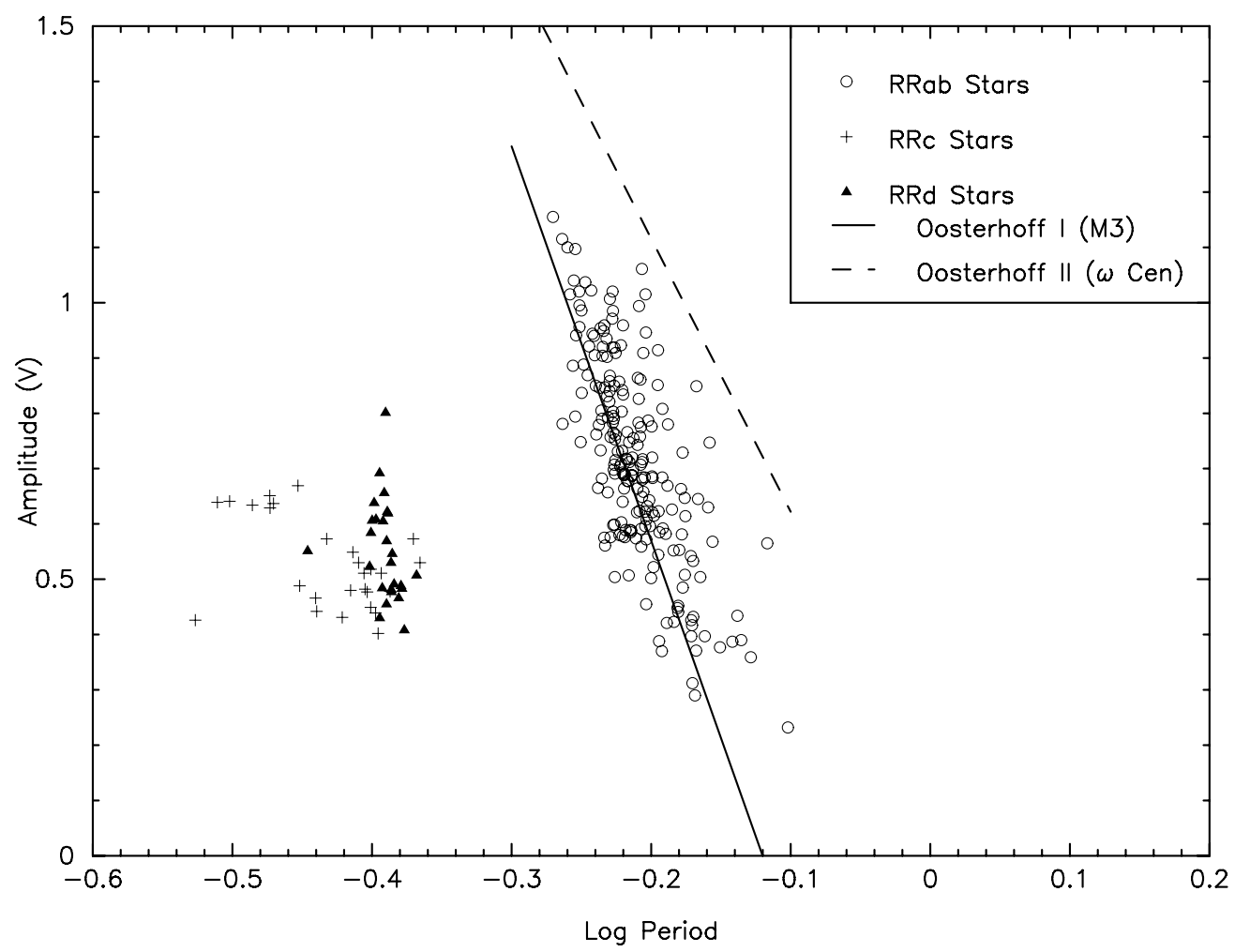

Figure 14. Period-amplitude diagram of the Draco RRL stars. The Clement \& Rowe (2000) relations for Oosterhoff I and Oosterhoff II are plotted to guide the eye for the Oosterhoff classification.

According to the $\Lambda$-cold dark matter hierarchical model, the outer halo has been assembled partly due to the accretion of objects like the Local Group dwarf galaxy population. However, almost no globular clusters of the Galaxy have Oosterhoff intermediate properties. Nor does the field RRL population of the halo resemble that of Draco (see, for example Kinemuchi et al. 2006). Thus, it seems likely that systems exactly like Draco could not have been main contributors to building the halo. In addition, Shetrone et al. (2001b) and Pritzl et al. (2005) found that the patterns of elemental abundances in the dSph galaxies were distinct from those in globular clusters and halo field stars. However, Bellazzini et al. (2002) argued that objects like Draco could still be considered as a building block if we consider that the accretion may have occurred early in the star-formation history of the dwarf galaxy or during an early episode of gas stripping by the Galaxy. Our findings with Draco at least imply that objects like this dwarf galaxy could not have contributed to the formation of the outer halo, even if it was accreted before the old population was formed in the dSph galaxy.

\section{SUMMARY}

We have presented the latest census of variable stars of the Draco dSph galaxy. We have found 81 new RRab stars, 8 new RRc stars, and 16 probable new RRd stars, thus bringing the total number of RRL stars of the different types known in Draco to $214 \mathrm{RRab}, 30 \mathrm{RRc}$, and $26 \mathrm{RRd}$. We have increased the number of ACs from five to nine. Draco cannot be clearly classified as an Oosterhoff I or an Oosterhoff II type system. Based upon the mean period of its RRab stars and their location in the period-amplitude diagram, Draco is Oosterhoff intermediate. Objects exactly like Draco are thus not likely to be important building blocks in forming the Galactic halo. We note, however, that the properties of the RRd stars in Draco are, with a single exception, similar to those of RRd stars in Oosterhoff II clusters.

The ACs in the Draco dSph galaxy show a possible dual $\mathrm{P}-\mathrm{L}$ relation stemming from the pulsational modes of the stars. However, with so few stars populating the first-overtone relation, we cannot say with certainty that two $\mathrm{P}-\mathrm{L}$ relation alternative is the only relation capable of describing the relationships of luminosity and period for Draco AC stars. In addition to the pulsating variable stars, we find two field eclipsing binary stars, 30 background QSOs, and 12 long-period variable stars.

This research used the facilities of the Canadian Astronomy Data Centre operated by the National Research Council of Canada with the support of the Canadian Space Agency. The identification of QSOs is based partly on spectra obtained with the Hydra multifiber spectrograph on the WIYN telescope at Kitt Peak National Observatory, National Optical Astronomical Observatories. NOAO is operated by the Association of Universities for Research in Astronomy, Inc., under cooperative agreement with the National Science Foundation. This research has made use of the USNOFS Image and Catalogue Archive operated by the United States Naval Observatory, Flagstaff Station (http://www.nofs.navy.mil/data/fchpix/).

H.A.S. acknowledges support from NSF grant AST 0607249. K.K. acknowledges support from NSF grant AST-0307778. The authors thank the referee, Gisella Clementini, for very useful comments and detailed suggestions for the overall improvement of this paper.

\section{REFERENCES}

Aaronson, M., Hodge, P. W., \& Olszewski, E. W. 1983, ApJ, 267, 271 Aaronson, M., Liebert, J., \& Stocke, J. 1982, ApJ, 254, 507 Aparicio, A., Carrera, R., \& Martínez-Delgado, D. 2001, AJ, 122, 2524 
Armandroff, T. E., Olszewski, E. W., \& Pryor, C. 1995, AJ, 110, 2131

Azzopardi, M., Lequeux, J., \& Westerlund, B. E. 1986, A\&A, 161, 232

Baade, W., \& Swope, H. H. 1961, AJ, 66, 300

Bellazzini, M., Ferraro, F. R., Origlia, L., Pancino, E., Monaco, L., \& Oliva, E. 2002, AJ, 124, 3222

Bonanos, A. Z., Stanek, K. Z., Szentgyorgyi, A. H., Sasselov, D. D., \& Bakos, G. Á. 2004, AJ, 127, 861

Bono, G., Caputo, F., Santolamazza, P., Cassisi, S., \& Piersimoni, A. 1997, AJ, 113,2209

Cacciari, C., \& Clementini, G. 2003, in Lecture Notes in Physics 635, Stellar Candles for the Extragalactic Distance Scale, ed. D. Alloin \& W. Gieren (Berlin: Springer), 105-122

Cacciari, C., Corwin, T. M., \& Carney, B. W. 2005, AJ, 129, 267

Cardelli, J. A., Clayton, G. C., \& Mathis, J. S. 1989, ApJ, 345, 245

Catelan, M. 2004, in ASP Conf. Ser. 310, Variable Stars in the Local Group (IAU Colloquium 193), ed. D. W. Kurtz \& K. R. Pollard (San Francisco, CA: ASP), 113

Catelan, M. 2005, arXiv:astro-ph/10507464

Clement, C. M., \& Rowe, J. 2000, AJ, 120, 2579

Clement, C. M., \& Shelton, I. 1999, ApJ, 515, L85

Clement, C. M., et al. 2001, AJ, 122, 2587

Dall'Ora, M., et al. 2003, AJ, 126, 197

Faria, D., Feltzing, S., Lundström, I., Gilmore, G., Wahlgren, G. M., Ardeberg, A., \& Linde, P. 2007, A\&A, 465, 357

Ferraz-Mello, S. 1981, AJ, 86, 619

Foster, G. 1995, AJ, 109, 1889

Goranskij, V. P. 1982, Astron. Tsirk., 1216, 5

Harris, H. C. 1987, in Lecture Notes in Physics 274, Stellar Pulsation, ed. A. N. Cox, W. M. Sparks, \& S. G. Starrfield (Berlin: Springer), 274-283

Hilker, M. 2000, A\&A, 355, 994

Honeycutt, R. K. 1992, PASP, 104, 435

Jurcsik, J. 1995, Acta Astron., 45, 653

Jurcsik, J., \& Kovacs, G. 1996, A\&A, 312, 111

Kinemuchi, K., Smith, H. A., Woźniak, P. R., \& McKay, T. A. 2006, AJ, 132, 1202

Kinman, T. D., Suntzeff, N. B., \& Kraft, R. P. 1994, AJ, 108, 1722

Kolenberg, K., et al. 2006, A\&A, 459, 577

Kovacs, G., \& Kanbur, S. M. 1998, MNRAS, 295, 834

Landolt, A. U. 1992, AJ, 104, 340

Lee, Y., Demarque, P., \& Zinn, R. 1990, ApJ, 350, 155

Lenz, P., \& Breger, M. 2005, Commun. Asteroseismol., 146, 53

Margon, B., et al. 2002, AJ, 124, 1651

Matthews, T. A., \& Sandage, A. R. 1963, ApJ, 138, 30

Momany, Y., Held, E. V., Saviane, I., Zaggia, S., Rizzi, L., \& Gullieuszik, M. 2007, A\&A, 468, 973
Nemec, J. M. 1985a, AJ, 90, 204

Nemec, J. M. 1985b, AJ, 90, 240

Nemec, J., Mendes de Oliveira, C., \& Wehlau, A. 1988, in ASP Conf. Ser. 4

The Extragalactic Distance Scale, ed. S. van den Bergh \& C. J. Pritchet (San Francisco, CA: ASP), 180

Nemec, J. M., Nemec, A. F. L., \& Lutz, T. E. 1994, AJ, 108, 222

Norris, J., \& Zinn, R. 1975, ApJ, 202, 335

Olszewski, E. W., Aaronson, M. \& Hill, J. M. 1995, AJ, 110, 2120

Oosterhoff, P. T. 1939, Observatory, 62, 104

Petersen, J. O. 1973, A\&A, 27, 89

Pritzl, B. J., Armandroff, T. E., Jacoby, G. H., \& Da Costa, G. S. 2002a, AJ, 124, 1464

Pritzl, B. J., Smith, H. A., Catelan, M., \& Sweigart, A. V. 2002b, AJ, 124,949

Pritzl, B. J., Venn, K. A., \& Irwin, M. 2005, AJ, 130, 2140

Purdue, P., Silbermann, N. A., Gay, P., \& Smith, H. A. 1995, AJ, 110, 1712

Reimann, J. D. 1994, PhD thesis, Univ. California at Berkeley

Renzini, A., \& Fusi Pecci, F. 1988, ARA\&A, 26, 199

Rood, R. T., \& Crocker, D. A. 1989, in IAU Colloq. 111, The Use of Pulsating Stars in Fundamental Problems of Astronomy, ed. E. G. Schmidt (Dordrecht: Kluwer), 103-119

Sandage, A. 1958, Ric. Astron., 5, 41

Sandage, A., Katem, B., \& Sandage, M. 1981, ApJS, 46, 41

Schlegel, D. J., Finkbeiner, D. P., \& Davis, M. 1998, ApJ, 500, 525

Schmidt, M. 1963, Nature, 197, 1040

Schneider, D. P., et al. 2005, AJ, 130, 367

Shetrone, M. D., Côté, P., \& Sargent, W. L. W. 2001a, ApJ, 548, 592

Shetrone, M. D., Côté, P., \& Stetson, P. B. 2001b, PASP, 113, 1122

Simon, N. R. 1979, A\&A, 74, 30

Simon, N. R., \& Teays, T. J. 1982, ApJ, 261, 586

Smith, H. A. 1995, RR Lyrae Stars, Cambridge Astrophysics Series (Cambridge: Cambridge Univ. Press)

Stellingwerf, R. F. 1978, ApJ, 224, 953

Stetson, P. B. 1980, AJ, 85, 387

Stetson, P. B. 1987, PASP, 99, 191

Stetson, P. B. 1994, PASP, 106, 250

Stothers, R. B. 2006, ApJ, 652, 643

Walker, A. R. 1994, AJ, 108, 555

Walker, A. R., \& Nemec, J. M. 1996, AJ, 112, 2026

Wallerstein, G., \& Cox, A. N. 1984, PASP, 96, 677

Wehlau, A., Bohlender, D., \& Demers, S. 1986, PASP, 98, 872

Welch, D. L., \& Stetson, P. B. 1993, AJ, 105, 1813

Zinn, R. 1978, ApJ, 225, 790

Zinn, R., \& Dahn, C. C. 1976, AJ, 81, 527

Zinn, R., \& Searle, L. 1976, ApJ, 209, 734 